\title{
Chemical interactions and configurational disorder in silicate melts
}

\author{
Giulio Ottonello \\ Laboratorio di Geochimica, Dipartimento per lo Studio del Territorio e delle sue Risorse (DipTeRis), \\ Università degli Studi di Genova, Italy
}

\begin{abstract}
The Thermodynamics of quasi-chemical and polymeric models are briefly reviewed. It is shown that the two classes are mutually consistent, and that opportune conversion of the existing quasi-chemical parameterization of binary interactions in $\mathrm{MO}-\mathrm{SiO}_{2}$ joins to polymeric models may be afforded without substantial loss of precision. It is then shown that polymeric models are extremely useful in deciphering the structural and reactive properties of silicate melts and glasses. They not only allow the Lux-Flood character of the dissolved oxides to be established, but also discriminate subordinate strain energy contributions to the Gibbs free energy of mixing from the dominant chemical interaction terms. This discrimination means that important information on the short-, medium- and long-range periodicity of this class of substances can be retrieved from thermodynamic analysis. Lastly, it is suggested that an important step forward in deciphering the complex topology of the inhomogeneity ranges observed at high $\mathrm{SiO}_{2}$ content can be performed by applying SCMF theory and, particularly, Matsen-Schick spectral analysis, hitherto applied only to rubberlike materials.
\end{abstract}

Key words silicate melts - structure - entropy - unmixing

\section{Introduction}

In Bernal's words (Bernal, 1959), a liquid is an «homogeneous, coherent and essentially irregular assemblage of molecules containing no crystalline regions or holes large enough to admit another molecule». This acceptance of a liquid structure is particularly in line with the Random Network Model (RNM) of Zachariasen (1932, 1933) and Warren (1933) for silica melts and glasses: silicate tetrahedrals linked through corners without any long-range periodicity. Already in the 1960s, Evans and King (1966) had physi-

Mailing address: Dr. Giulio Ottonello, Laboratorio di Geochimica, Dipartimento per lo Studio del Territorio e delle sue Risorse (DipTeRis), Università degli Studi di Genova, Corso Europa 26, 16132 Genova, Italy; e-mail: giotto@dipteris.unige.it cally built up an RNM composed of Corning Glassworks tetrapods linked by bent springs, the angles of the bends having a Montecarlo-generated Gaussian distribution centered at $163^{\circ}$. The measured radial distribution of atoms around the silicon and oxygen reference atoms of the physical model appeared to be in good agreement with existing neutron and $X$-ray scattering data (Warren et al., 1936; Warren, 1937; Carraro, 1964). More than half a century of experimental research essentially confirmed the first illuminating views of Zachariasen, Warren and Bernal, and nowadays we may basically state the following:

- The structure of silicate melts and glasses has elements of long-range randomness coexisting with short- and medium-range order (Gaskell et al., 1991).

- The short-range order is dominated by the structure of the $\mathrm{SiO}_{4}$ rigid unit (Greaves et al., 1981), although the distance beyond which the radial distribution function becomes indistinguishable from the $4 \pi r^{2} \rho_{0}$ function, representing homogeneous distribution of electron density, shrinks progressively with the introduction 
of alkalis («network modifiers»; see later) in the system (Waseda and Suito, 1977), which is indicative of significant break-up of the random network.
- There are no systematic structural differences between silicate melts and glasses of identical composition, and the effects of thermal expansion are virtually negligible on both

Table I. Structural data for binary silicate melts and glasses (from Brown et al., 1995). Interionic distance $r_{i j}(A)$ approximated to $\pm 0.01 \mathrm{~A}$; coordination number $N_{i j}$ approximated to \pm 0.3 atoms; root mean square atomic displacement $\Delta r_{i j}{ }^{1 / 2}$ approximated to \pm 0.005 .

\begin{tabular}{|c|c|c|c|c|c|c|c|c|c|}
\hline \multirow[t]{2}{*}{ Join } & \multirow[t]{2}{*}{$T\left({ }^{\circ} \mathrm{C}\right)$} & \multirow[t]{2}{*}{ Pair } & \multicolumn{3}{|c|}{$X$-ray (molten state) } & \multicolumn{3}{|c|}{$X$-ray (glassy state) } & \multirow[t]{2}{*}{ Reference } \\
\hline & & & $r_{i j}(A)$ & $N_{i j}$ (atoms) & $\Delta r_{i j}^{1 / 2}$ & $r_{i j}(A)$ & $N_{i j}$ (atoms) & $\Delta r_{i j}^{1 / 2}$ & \\
\hline \multirow[t]{3}{*}{ Pure $\mathrm{SiO}_{2}$} & \multirow[t]{3}{*}{1750} & $\mathrm{Si}-\mathrm{O}$ & 1.62 & 3.8 & 0.096 & 1.62 & 3.9 & 0.087 & (1) \\
\hline & & $\mathrm{O}-\mathrm{O}$ & 2.65 & 5.6 & 0.124 & 2.65 & 5.5 & 0.102 & (1) \\
\hline & & $\mathrm{Si}-\mathrm{Si}$ & 3.12 & 3.9 & 0.187 & 3.11 & 3.9 & 0.141 & (1) \\
\hline \multirow{4}{*}{$\begin{array}{l}0.33 \mathrm{Li}_{2} \mathrm{O}- \\
-0.67 \mathrm{SiO}_{2}\end{array}$} & \multirow[t]{4}{*}{1150} & $\mathrm{Si}-\mathrm{O}$ & 1.61 & 3.8 & 0.117 & 1.62 & 3.7 & 0.090 & (2) \\
\hline & & $\mathrm{Li}-\mathrm{O}$ & 2.08 & 4.1 & 0.131 & 2.07 & 3.8 & 0.095 & (2) \\
\hline & & $\mathrm{O}-\mathrm{O}$ & 2.66 & 5.5 & 0.195 & 2.65 & 5.6 & 0.101 & (2) \\
\hline & & $\mathrm{Si}-\mathrm{Si}$ & 3.13 & 3.8 & 0.260 & 3.13 & 3.8 & 0.143 & (2) \\
\hline \multirow{4}{*}{$\begin{array}{l}0.33 \mathrm{Na}_{2} \mathrm{O}- \\
-0.67 \mathrm{SiO}_{2}\end{array}$} & \multirow[t]{4}{*}{1000} & $\mathrm{Si}-\mathrm{O}$ & 1.62 & 4.1 & 0.095 & 1.62 & 4.0 & 0.086 & (2) \\
\hline & & $\mathrm{Na}-\mathrm{O}$ & 2.36 & 5.9 & 0.151 & 2.36 & 5.8 & 0.101 & (2) \\
\hline & & $\mathrm{O}-\mathrm{O}$ & 2.66 & 5.6 & 0.202 & 2.65 & 5.2 & 0.112 & (2) \\
\hline & & $\mathrm{Si}-\mathrm{Si}$ & 3.20 & 3.8 & 0.279 & 3.21 & 3.6 & 0.146 & (2) \\
\hline \multirow{4}{*}{$\begin{array}{l}0.33 \mathrm{~K}_{2} \mathrm{O}- \\
-0.67 \mathrm{SiO}_{2}\end{array}$} & \multirow[t]{4}{*}{1100} & $\mathrm{Si}-\mathrm{O}$ & 1.62 & 3.9 & 0.124 & 1.62 & 3.8 & 0.086 & (2) \\
\hline & & K-O & 2.66 & $13.0^{*}$ & $0.182^{*}$ & 2.65 & $13.2^{*}$ & $0.120^{*}$ & (2) \\
\hline & & $\mathrm{O}-\mathrm{O}$ & 2.66 & & & 2.65 & & & (2) \\
\hline & & $\mathrm{Si}-\mathrm{Si}$ & 3.23 & 3.7 & 0.257 & 3.23 & 3.5 & 0.154 & (2) \\
\hline \multirow{4}{*}{$\begin{array}{l}0.50 \mathrm{MgO}- \\
-0.50 \mathrm{SiO}_{2}\end{array}$} & \multirow[t]{4}{*}{1700} & $\mathrm{Si}-\mathrm{O}$ & 1.62 & 3.9 & 0.109 & 1.63 & 3.7 & 0.096 & (3) \\
\hline & & $\mathrm{Mg}-\mathrm{O}$ & 2.12 & 4.3 & 0.151 & 2.14 & 4.6 & 0.108 & (3) \\
\hline & & $\mathrm{O}-\mathrm{O}$ & 2.65 & 5.4 & 0.215 & 2.65 & 5.7 & 0.151 & (3) \\
\hline & & $\mathrm{Si}-\mathrm{Si}$ & 3.16 & 3.3 & 0.282 & 3.15 & 3.4 & 0.213 & (3) \\
\hline \multirow{4}{*}{$\begin{array}{c}0.50 \mathrm{CaO}- \\
-0.50 \mathrm{SiO}_{2}\end{array}$} & \multirow[t]{4}{*}{1600} & $\mathrm{Si}-\mathrm{O}$ & 1.61 & 3.9 & 0.127 & 1.63 & 3.8 & 0.109 & (3) \\
\hline & & $\mathrm{Ca}-\mathrm{O}$ & 2.35 & 5.9 & 0.171 & 2.43 & 5.9 & 0.125 & (3) \\
\hline & & $\mathrm{O}-\mathrm{O}$ & 2.67 & 5.2 & 0.206 & 2.66 & 5.5 & 0.183 & (3) \\
\hline & & $\mathrm{Si}-\mathrm{Si}$ & 3.20 & 3.1 & 0.264 & 3.23 & 3.4 & 0.199 & (3) \\
\hline \multirow{4}{*}{$\begin{array}{l}0.66 \mathrm{FeO}- \\
-0.33 \mathrm{SiO}_{2} \#\end{array}$} & \multirow[t]{4}{*}{1400} & $\mathrm{Si}-\mathrm{O}$ & 1.62 & 3.9 & 0.147 & - & - & - & (4) \\
\hline & & $\mathrm{Fe}-\mathrm{O}$ & 2.05 & 3.9 & 0.214 & - & - & - & (4) \\
\hline & & $\mathrm{O}-\mathrm{O}$ & - & - & - & - & - & - & (4) \\
\hline & & $\mathrm{Si}-\mathrm{Si}$ & 3.27 & 3.1 & 0.302 & - & - & - & (4) \\
\hline
\end{tabular}

(1) Waseda and Toguri (1990); (2) Waseda and Suito (1977); (3) Waseda and Toguri (1977); (4) Waseda and Toguri (1978); ${ }^{*}$ pair correlations for K-O and O-O overlap, so that coordination numbers and peak widths could not be determined independently; \# O-O pair correlation overlaps with $\mathrm{Fe}-\mathrm{Fe}$ correlations, thus O-O distance could not be determined independently. 
mean structure and single interionic distances (see, for this purpose, the review article of Brown et al., 1995, and references therein).

Table I lists some important $X$-ray scattering data concerning the structure of binary silicate melts collected by Brown et al. (1995) and essentially in line with the above three points. Table II, based on the extensive compilation by Brown et al. (1995), lists concisely the mean coordination numbers experimentally observed for the various cations in melts and glasses. Although a generalized increase in the coordination number of the central cation is observed with the increase in the Lux-Flood basicity of the corresponding oxide, the scatter in $\mathrm{CN}$ observed for basic components is a clear indication that network modifiers «adapt» themselves to the geometry established by the much stronger (and essentially covalent) bond of network formers, and not vice versa. In this light, it is difficult to agree with the hypothesis of Gaskell et al. (1991) that compositionally similar pyroxene or pyroxenoid glasses may be represented as parallel random-fractal sheets of interconnected $\mathrm{CaO}_{6}$ octahedra interleaved by $\mathrm{Si}$ atoms (occupying intersitial tetrahedral sites linked in infinite chains). More probably, in the present author's opinion, the structure of silicate melts or glasses locally resemble that of all-Si zeolites, in which «cages» of variable coordination number are determined by the relative symmetry-determining arrangements of $\mathrm{SiO}_{4}$ monomeric units.

Despite this topological controversy, it is quite evident that conformational disorder does exist in silicate melts and glasses. Exactly how thermodynamics can account for this conformational disorder may be purely phenomenological (as in subregular models and polynomial expansions) or may be based on more sophisticated physical models (as in the Gaussian Random Walk method; see later). In any case, to be satisfactory, the thermodynamic model must not only reproduce the expected reactive properties of the substance, but also the inhomogeneity ranges often observed at high $\mathrm{SiO}_{2}$ content.

We discuss below the informational content arising from sound thermodynamic treatment of silicate melt energetics, and propose some
Table II. $P=1$ bar mean M-O coordination number of cations in silicate melts and glasses. Listed figures are largely based on extensive compilation of Brown et al. (1995). Allocation among acidic, amphotheric and basic oxides after Ottonello and Moretti (2004) and this work (\#).

\begin{tabular}{|c|c|c|c|c|}
\hline$M$ & Acidic & Amphotheric & Basic & Notes \\
\hline $\mathrm{Si}$ & 4 & & & \\
\hline Ge\# & 4 & & & \\
\hline $\mathrm{Al}$ & 4 & & & $\begin{array}{l}\text { In Na-Al. } \\
\text { Si-O melts }\end{array}$ \\
\hline $\mathrm{Al}$ & $5.5-6$ & & & $\begin{array}{c}\text { In pure } \\
\mathrm{Al}_{2} \mathrm{O}_{3} \text { melt. }\end{array}$ \\
\hline FeIII & & 4 & & \\
\hline $\mathrm{Ti}$ & & $4-5-6$ & & \\
\hline $\mathrm{Zr}$ & & $6-8$ & & \\
\hline $\mathrm{Ni}$ & & $4-5-6$ & & \\
\hline $\mathrm{Li} \#$ & & & 4 & \\
\hline $\mathrm{Na}$ & & & $5-6$ & \\
\hline $\mathrm{K}$ & & & $7-8$ & \\
\hline $\mathrm{Rb} \#$ & & & 8 & \\
\hline Cs & & & 8 & \\
\hline $\mathrm{Ca}$ & & & $4-6-7-8$ & \\
\hline $\mathrm{Mg}$ & & & $4-5-6$ & \\
\hline FeII & & & $4-5-6$ & \\
\hline $\mathrm{Mn}$ & & & $4-5-6$ & \\
\hline $\mathrm{Pb}$ & & & 8 & \\
\hline
\end{tabular}

conceptual guidelines to be followed in the near future, in order to decipher the complex features of the inhomogeneity ranges observed at high $\mathrm{SiO}_{2}$ content along simple $\mathrm{MO}-\mathrm{SiO}_{2}$ binaries, and also in chemically complex systems.

\section{Configurational disorder in quasi-chemical models}

Guggenheim's quasi-chemical model (Guggenheim, 1935; Fowler and Guggenheim, 1939) assumes that, in a binary system with components 1 and 2, «particles» 1 and 2 mix substitutionally in a quasi-lattice obeying short-range ordering dictated by the equilibrium 


$$
[1-1]+[2-2] \Longleftrightarrow 2[1-2] .
$$

The molar enthalpy change associated with equilibrium (2.1) is expressed in terms of pair bond energies $\varepsilon_{i j}$

$$
\omega=\frac{N Z}{2}\left(2 \varepsilon_{12}-\varepsilon_{11}-\varepsilon_{22}\right)
$$

where $N$ is the total number of atoms and $Z$ is the coordination number.

The non-configurational part of the molar entropy is also given in terms of bond contributions $\sigma_{i j}$ (non-configurational entropy of the $i j$ pair bond)

$$
\omega=\frac{N Z}{2}\left(2 \eta_{12}-\eta_{11}-\eta_{22}\right)
$$

If $X_{i j}$ denotes the molar fraction of the $i j$ particle with respect to the total of the particles formed in the system, since each atom $i$ is bonded to $Z_{j}$ neighbors, it follows from mass balance that

$$
\frac{X_{12}}{2}=\left(X_{1}-X_{11}\right)=\left(X_{2}-X_{22}\right) .
$$

The enthalpy of mixing then becomes

$$
H_{\text {mixing }}=\frac{X_{12}}{2} \omega
$$

the non-configurational (excess) entropy of mixing is

$$
S_{\text {mixing, non-conf }}=\frac{X_{12}}{2} \eta
$$

and the configurational (ideal + excess) entropy of mixing is

$$
\begin{aligned}
& S_{\text {mixing, conf }}=-R\left(X_{1} \ln X_{1}+X_{2} \ln X_{2}\right)-\frac{R Z}{2} . \\
& \left(X_{11} \ln \frac{X_{11}}{X_{1}^{2}}+X_{22} \ln \frac{X_{22}}{X_{2}^{2}}+X_{12} \ln \frac{X_{12}}{2 X_{1} X_{2}}\right)
\end{aligned}
$$

(a)

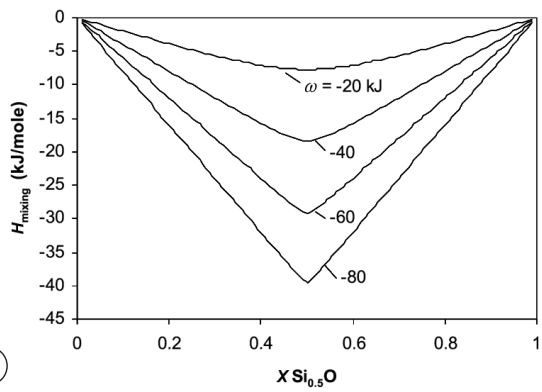

(b)
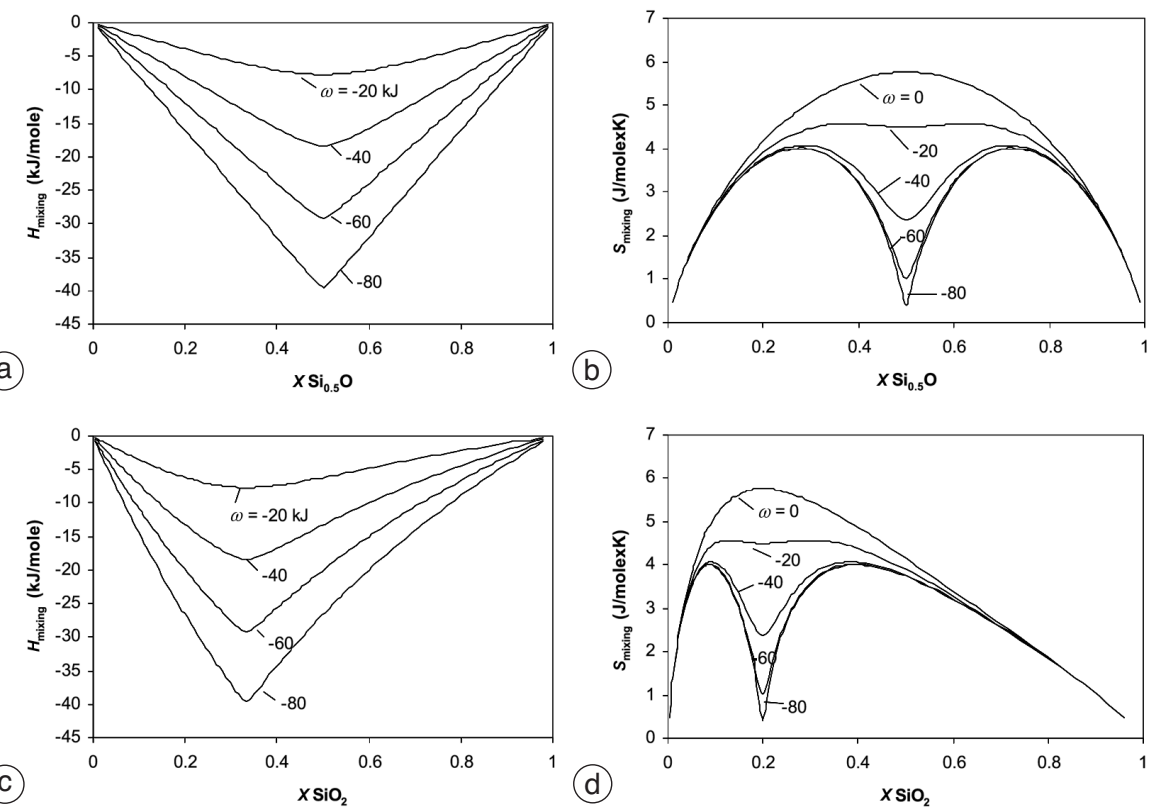

(d)

Fig. 1a-d. Integral enthalpy of mixing and integral entropy of mixing of quasi-chemical model for different values of interaction parameter $\omega$ along $\mathrm{MO}-\mathrm{Si}_{0.5} \mathrm{O}_{2}$ join ((a) and (b), respectively). Below: if usual molar notation (i.e., component $\mathrm{SiO}_{2}$ instead of $\mathrm{Si}_{0.5} \mathrm{O}$ ) is adopted and parameter $\omega$ is kept unvaried, symmetricity about intermediate composition is lost ((c) and (d) respectively). 
because when the mixture is completely random, $X_{11}=X_{1}^{2}, X_{22}=X_{2}^{2}$ and $X_{12}=X_{1} X_{2}$.

The equilibrium concentrations of the various pairs obey the Gibbs free energy minimization principle

$$
\frac{\partial G_{\text {mixing }}}{\partial X_{12}}=\frac{\partial\left(H_{\text {mixing }}-T S_{\text {mixing }}\right)}{\partial X_{12}}=0 .
$$

This, through opportune expansions in terms of eqs. ((2.5) to (2.7)), gives

$$
\frac{X_{12}^{2}}{X_{11} X_{22}}=4 \exp \left[\frac{-2(\omega-\eta T)}{Z R T}\right] \text {. }
$$

The name «quasi-chemical» assigned to the model stems from the fact that the term on the right in eq. (2.9) reduces to a constant, for constant values of $\omega$ and $\eta$, thus resembling the notation of a chemical reaction among ideal [1-1], [2-2] reactants and [1-2] product.

Equation (2.9) yields (Pelton and Blander, 1986)

$$
\frac{X_{12}}{2}=\frac{2 X_{1} X_{2}}{1+\xi}
$$

where

$$
\xi=\left\{1+4 X_{1} X_{2}\left[\exp \left(\frac{2(\omega-\eta T)}{Z R T}\right)-1\right]\right\}^{\frac{1}{2}}
$$

The model is resolved first in terms of equilibrium distribution of particles (eqs. (2.10), (2.11) and (2.4)) and then in terms of enthalpy and entropy (eq. (2.5) and eqs. ((2.6) to (2.7)), respectively).

The computed enthalpic and entropic terms have a typical $V$ - and $M$-shaped conformation, the more pronounced as the value assigned to $\omega$ becomes more negative. The quasi-chemical enthalpy of mixing and entropy of mixing curves computed with $Z=2, \eta=0$ and various $\omega$ are shown for comparison in fig. 1a,b, respectively, for a $\mathrm{MO}-\mathrm{Si}_{0.5} \mathrm{O}$ system. When $\omega$ and $\eta$ are set at zero the mixture is obviously ideal and, when $\eta=0$ and $X_{12}=2 X_{1} X_{2}$, the mixture is strictly regular. In all cases, mixtures are always symmetric in the compositional space of interest.

\subsection{Modified quasi-chemical approach}

The symmetricity about intermediate composition was long regarded as a severe limitation to the application of the quasi-chemical model to silicate melts and slags which invariantly exhibit maximum ordering about the composition $X_{\mathrm{MO}}=2 / 3$ in the $\mathrm{MO}-\mathrm{SiO}_{2}$ chemical space. In order to shift the maximum ordering condition depicted by the quasi-chemical model to the condition actually observed in MO$\mathrm{SiO}_{2}$ melts, Pelton and Blander (1986) proposed substituting true molar fractions $X_{1}, X_{2}$ with «equivalent fractions» $Y_{1}, Y_{2}$ so that

$$
\begin{gathered}
Y_{1}=\frac{b_{1} X_{1}}{b_{1} X_{1}+b_{2} X_{2}} \\
Y_{2}=\frac{b_{2} X_{2}}{b_{1} X_{1}+b_{2} X_{2}} .
\end{gathered}
$$

Coefficients $b_{1}$ and $b_{2}$ are chosen in such a way as to have $b_{1} /\left(b_{1}+b_{2}\right)=1 / 3$ and $b_{2} /\left(b_{1}+b_{2}\right)=2 / 3$. Clearly, when $X_{1}=2 / 3$ and $X_{2}=1 / 3$, we have $Y_{1}=Y_{2}=0.5$.

Through this substitution it follows that, instead of eq. (2.4), the following equation holds (Pelton and Blander, 1986):

$$
\frac{X_{12}}{2}=\left(Y_{1}-X_{11}\right)=\left(Y_{2}-X_{22}\right) \text {. }
$$

The molar enthalpy of mixing is now (Pelton and Blander, 1986)

$$
H_{\text {mixing }}=\frac{X_{12}}{2}\left(b_{1} X_{1}+b_{2} X_{2}\right) \omega
$$

the non-configurational (excess) entropy of mixing is

$$
S_{\text {mixing, non-conf }}=\frac{X_{12}}{2}\left(b_{1} X_{1}+b_{2} X_{2}\right) \eta
$$

and the configurational (ideal + excess) entropy of mixing is

$$
\begin{aligned}
& S_{\text {mixing, conf }}=-R\left(X_{1} \ln X_{1}+X_{2} \ln X_{2}\right)-\frac{R Z}{2} . \\
& \left(X_{11} \ln \frac{X_{11}}{Y_{1}^{2}}+X_{22} \ln \frac{X_{22}}{Y_{2}^{2}}+X_{12} \ln \frac{X_{12}}{2 Y_{1} Y_{2}}\right) . \\
& \cdot\left(b_{1} X_{1}+b_{2} X_{2}\right) .
\end{aligned}
$$


Although whatever $b_{1}, b_{2}$ couple exists, so that $b_{1} / b_{2}=1 / 2$ results in $Y_{1} / Y_{2}=1 / 2$, application of the additional condition that configurational entropy must attain zero when $\omega=-\infty$ yields $b_{1}=0.6887, b_{2}=1.3774$ for binary $\mathrm{MO}-\mathrm{SiO}_{2}$ melts with maximum ordering about $X_{\mathrm{MO}}=2 / 3$ ( $c f$. Pelton and Blander, 1986, for details).

Lastly, in order to assign more computational elasticity to the model, parameters $\omega$ and $\eta$ are expanded as polynomial functions of equivalent fractions $Y_{1}, Y_{2}$, i.e.

$$
\begin{aligned}
& \omega=\omega_{0}+\omega_{1} Y_{2}+\omega_{2} Y_{2}^{2}+\ldots \omega_{n} Y_{2}^{n} \\
& \eta=\eta_{0}+\eta_{1} Y_{2}+\eta_{2} Y_{2}^{2}+\ldots \eta_{n} Y_{2}^{n} .
\end{aligned}
$$

Actually, the asymmetricity of $\mathrm{MO}-\mathrm{SiO}_{2}$ joins is due to the difference in the amounts of oxygen in the two end-member components. If we adopt $\mathrm{Si}_{0.5} \mathrm{O}$ instead of $\mathrm{SiO}_{2}$ as end-member, maximum ordering will be observed at $X_{\mathrm{MO}}=0.5$, and no readjustment in terms of equivalent fractions will be necessary. Obviously, if we wish to rep-

Table III. Chemical interaction parameters of modified-quasi-chemical model for binary silicate melts (after Pelton et al., 1995).

\begin{tabular}{lcccrrrrrrrr}
\hline \hline $\mathrm{AO}$ & $\mathrm{BO}$ & $b_{1}$ & \multicolumn{1}{c}{$b_{2}$} & \multicolumn{1}{c}{$\omega_{0}$} & \multicolumn{1}{c}{$\omega_{1}$} & $\omega_{2}$ & $\omega_{3}$ & $\omega_{4}$ & $\omega_{5}$ & $\omega_{6}$ & $\omega_{7}$ \\
\hline $\mathrm{AlO}_{1.5}$ & $\mathrm{SiO}_{2}$ & 2.0661 & 2.7548 & 4800. & 0. & 0.100784. & 0. & -142068. & 0. & 78571. \\
$\mathrm{BO}_{1.5}$ & $\mathrm{SiO}_{2}$ & 2.0661 & 2.7548 & 16958. & -32531. & 0. & 0. & 0. & 0. & 0. & 0. \\
$\mathrm{CaO}$ & $\mathrm{SiO}_{2}$ & 1.3774 & 2.7548 & -158218. & -37932. & 0. & 0. & 0. & -90148. & 0. & 439893. \\
$\mathrm{KO}_{0.5}$ & $\mathrm{SiO}_{2}$ & 0.6887 & 2.7548 & -409986. & 0. & 0. & 0. & 0. & 0. & -1647688. & 1593677. \\
$\mathrm{MgO}$ & $\mathrm{SiO}_{2}$ & 1.3774 & 2.7548 & -86090. & -4874. & 0. & 0. & 0. & 0. & 0. & 328109. \\
$\mathrm{MnO}$ & $\mathrm{SiO}_{2}$ & 1.3774 & 2.7548 & -79956. & 0. & 0. & 0. & 0. & 0. & 0. & 228819. \\
$\mathrm{NaO}$ & $\mathrm{SiO}_{2}$ & 0.6887 & 2.7548 & -114344. & -381595. & 0. & 0. & 0. & 0. & 0. & 123010. \\
$\mathrm{NiO}$ & $\mathrm{SiO}_{2}$ & 1.3774 & 2.7548 & 29169. & 0. & 0. & 0. & 0. & 0. & 0. & 509783. \\
$\mathrm{PbO}$ & $\mathrm{SiO}_{2}$ & 1.3774 & 2.7548 & -25430. & 0. & 0. & 0. & 0. & 0. & -245806. & 310959. \\
$\mathrm{SiO}$ & $\mathrm{TiO}_{2}$ & 2.7548 & 2.7548 & 28847. & 52091. & 0. & -44484. & 0. & 0. & 0. & 0. \\
$\mathrm{ZnO}$ & $\mathrm{SiO}_{2}$ & 1.3774 & 2.7548 & -124741. & 129292. & 15989. & 0. & 0. & 0. & 0. & 98990. \\
$\mathrm{ZrO}_{2}$ & $\mathrm{SiO}_{2}$ & 2.7548 & 2.7548 & 4184. & 40585. & 0. & 0. & 0. & 0. & 0. & -11715. \\
\hline
\end{tabular}

Table IV. Non-configurational excess entropy parameters of modified-quasi-chemical model for binary silicate melts (after Pelton et al., 1995).

\begin{tabular}{lcccrrrrrrrrr}
\hline \hline $\mathrm{AO}$ & $\mathrm{BO}$ & $b_{1}$ & \multicolumn{1}{c}{$b_{2}$} & \multicolumn{1}{c}{$\eta_{0}$} & \multicolumn{1}{c}{$\eta_{1}$} & $\eta_{2}$ & & $\eta_{3}$ & $\eta_{4}$ & $\eta_{5}$ & $\eta_{6}$ & $\eta_{7}$ \\
\hline $\mathrm{AlO}_{1.5}$ & $\mathrm{SiO}_{2}$ & 2.0661 & 2.7548 & 0. & 0. & 0. & 0. & 0. & 0. & 0. & 0. \\
$\mathrm{BO}_{1.5}$ & $\mathrm{SiO}_{2}$ & 2.0661 & 2.7548 & 0. & -25.104 & 0. & 0. & 0. & 0. & 0. & 0. \\
$\mathrm{CaO}$ & $\mathrm{SiO}_{2}$ & 1.3774 & 2.7548 & -19.456 & 0. & 0. & 0. & 0. & 0. & 0. & 0. \\
$\mathrm{KO}_{0.5}$ & $\mathrm{SiO}_{2}$ & 0.6887 & 2.7548 & -58.576 & 0. & 0. & 0. & 0. & 0. & 0. & 0. \\
$\mathrm{MgO}$ & $\mathrm{SiO}_{2}$ & 1.3774 & 2.7548 & 0. & -37.656 & 0. & 0. & 0. & 0. & 0. & 125.52 \\
$\mathrm{MnO}$ & $\mathrm{SiO}_{2}$ & 1.3774 & 2.7548 & -20.92 & 0. & 0. & 0. & 0. & 0. & 0. & 62.76 \\
$\mathrm{NaO} 0.5$ & $\mathrm{SiO}_{2}$ & 0.6887 & 2.7548 & -43.932 & 0. & 0. & 0. & 0. & 0. & 0. & -20.92 \\
$\mathrm{NiO}$ & $\mathrm{SiO}_{2}$ & 1.3774 & 2.7548 & 0. & 0. & 0. & 0. & 0. & 0. & 0. & 125.52 \\
$\mathrm{PbO}$ & $\mathrm{SiO}_{2}$ & 1.3774 & 2.7548 & 0. & 0. & 0. & 0. & 0. & 0. & 0. & 12.97 \\
$\mathrm{SiO}$ & $\mathrm{TiO}_{2}$ & 2.7548 & 2.7548 & 0. & 0. & 0. & 0. & 0. & 0. & 0. & 0. \\
$\mathrm{ZnO}_{2}$ & $\mathrm{SiO}_{2}$ & 1.3774 & 2.7548 & -33.472 & 0. & 58.576 & 0. & 0. & 0. & 0. & 0. \\
$\mathrm{ZrO}_{2}$ & $\mathrm{SiO}_{2}$ & 2.7548 & 2.7548 & 0. & 0. & 0. & 0. & 0. & 0. & 0. & 0. \\
\hline
\end{tabular}


resent the chemical space of interest in terms of $\mathrm{MO}-\mathrm{SiO}_{2}$ components, then it is sufficient to consider that two moles of $\mathrm{Si}_{0.5} \mathrm{O}$ correspond to one mole of $\mathrm{SiO}_{2}$, the resulting molar fractions $X_{\mathrm{MO}}, X_{\mathrm{SiO}_{2}}$ are translated along the abscissa, and an apparent asymmetricity arises, as depicted in fig. $1 \mathrm{c}, \mathrm{d}$.

Tables III and IV list the extensive modified-quasi-chemical parameterization of binary silicate melts of Pelton et al. (1995). The listed parameters allow precise conformation of phase boundaries along the binary joins and are also consistent with observed unmixing phenomena.

\section{Configurational disorder in polymer models}

In polymeric models for silicate melts, it is postulated that, at each composition, for given values of $P$ and $T$, the melt is characterized by an equilibrium distribution of several ion species of oxygen, metal cations and ionic polymers of monomeric units $\mathrm{SiO}_{4}^{4-}$.

The charge balance of a polymerization reaction involving $\mathrm{SiO}_{4}^{4-}$ monomers may be formally described by a homogeneous reaction involving three forms of oxygen: singly bonded $\mathrm{O}^{-}$, doubly bonded (or «bridging oxygen») $\mathrm{O}^{0}$, and free oxygen $\mathrm{O}^{2-}$ (Fincham and Richardson, 1954)

$$
\underset{\text { melt }}{2 \mathrm{O}^{-}} \Longleftrightarrow \underset{\text { melt }}{\mathrm{O}^{0}}+\underset{\text { melt }}{\mathrm{O}^{2-}} .
$$

In fact, eq. (3.1) is similar to a reaction between monomers

$$
\mathrm{SiO}_{4}^{4-}+\mathrm{SiO}_{4}^{4-} \Longleftrightarrow \mathrm{Si}_{2} \mathrm{O}_{7}^{6-}+\mathrm{O}^{2-} .
$$

Polymer chemistry shows that, the larger the various polymers become, the more their reactivity is independent of the length of the polymer chains. This fact, known as «the principle of equal reactivity of co-condensing functional groups», has been verified in fused polyphosphate systems (which, for several properties, may be considered as analogous to silicate melts; $c f$. Fraser, 1977) with polymeric chains longer than $3 \mathrm{PO}_{4}^{4-}$ units (Meadowcroft and
Richardson, 1965; Cripps-Clark et al., 1974). Assuming this principle to be valid, equilibrium constant $K_{18}$

$$
K_{18}=\frac{\left(\mathrm{O}^{0}\right)\left(\mathrm{O}^{2-}\right)}{\left(\mathrm{O}^{-}\right)^{2}}
$$

(in which the terms in brackets represent the number of moles per unit mole of melt) is always representative of the polymerization process, independent of the effective length of the polymer chains.

\subsection{Toop-Samis model}

Toop and Samis (1962a,b) showed that, in a $\mathrm{MO}-\mathrm{SiO}_{2}$ binary melt, in which MO is the oxide of a basic cation completely dissociated in the melt, the total number of bonds per mole of melt is given by

$$
2\left(\mathrm{O}^{0}\right)\left(\mathrm{O}^{2-}\right)=4 N_{\mathrm{SiO}_{2}}
$$

where $N_{\mathrm{SiO}_{2}}$ are the moles of $\mathrm{SiO}_{2}$ in the MO$\mathrm{SiO}_{2}$ melt. The number of bridging oxygens is thus

$$
\left(\mathrm{O}^{\circ}\right)=\frac{4 N_{\mathrm{SiO}_{2}}-\left(\mathrm{O}^{-}\right)}{2} .
$$

Mass balance gives the number of moles of free oxygen per mole of melt

$$
\left(\mathrm{O}^{2-}\right)=\left(1-N_{\mathrm{SiO}_{2}}\right)-\frac{\left(\mathrm{O}^{-}\right)}{2} .
$$

where $\left(1-N_{\mathrm{SiO}_{2}}\right)$ are the moles of basic oxide. Equations ((3.3) to (3.6)) yield

$$
K_{18}=\frac{\left[4 N_{\mathrm{SiO}_{2}}-\left(\mathrm{O}^{-}\right)\right]\left[2-2 N_{\mathrm{SiO}_{2}}-\left(\mathrm{O}^{-}\right)\right]}{4\left(\mathrm{O}^{-}\right)^{2}} .
$$

$\left(\mathrm{O}^{-}\right)$is thus given by the quadratic equation

$$
\begin{aligned}
& \left(\mathrm{O}^{-}\right)^{2}\left(4 K_{18}-1\right)+\left(\mathrm{O}^{-}\right)\left(2+2 N_{\mathrm{SiO}_{2}}\right)+ \\
& +8 N_{\mathrm{SiO}_{2}}\left(N_{\mathrm{SiO}_{2}}-1\right)=0
\end{aligned}
$$

which may be solved for discrete values of $K_{18}$ and $N_{\mathrm{SiO}_{2}}$. 
The Gibbs free energy change involved in eq. (3.1) is

$$
\Delta G_{18}^{0}=-R T \ln K_{18} .
$$

Since two moles of $\mathrm{O}^{-}$produce one mole of $\mathrm{O}^{0}$ and one of $\mathrm{O}^{2-}$, the Gibbs free energy of mixing per mole of silicate melt is given by

$$
\Delta G_{\text {mixing }}=-\frac{\left(\mathrm{O}^{-}\right)}{2} R T \ln K_{18} .
$$

\subsection{Extended Polymeric Approach (EPA)}

Although the original Toop-Samis model is a powerful tool in deciphering the complex relations existing between chemical interaction and configurational disorder, there are two minor points that must be quantified, i.e. assessment of the low polymerization limit, and extension to the multicomponent field.

The usual expression of the thermodynamic activity of a component in mixture (Ottonello, 2001) yields

$$
a_{\mathrm{MO}}=\exp \left[\frac{\frac{\left(\mathrm{O}^{-}\right)}{2} R T \ln K_{18}+\left(1-X_{\mathrm{MO}}\right) \frac{\partial G_{\text {mixing }}}{\partial X_{\mathrm{MO}}}}{R T}\right] .
$$

As the mean number of monomers in the polymer chain is defined as

$$
\bar{\nu}_{\mathrm{Si}}=\left(\mathrm{SiO}_{2}\right) / \sum \text { anions }
$$

adopting Temkin model activities of the fused salts, it is quite evident that, along any binary join, we may pose

$$
\bar{\nu}_{\mathrm{Si}}=\frac{\left(\mathrm{SiO}_{2}\right)}{\frac{\left(\mathrm{O}^{2-}\right)}{a_{\mathrm{MO}, \text { binary }}}-\left(\mathrm{O}^{2-}\right)} .
$$

Although the domain of eq. (3.13) spans the entire compositional range, it is obvious that $\bar{\nu}_{\mathrm{Si}}$ can never be lower than one (i.e., a monomer) and, consequently, the limit of maximum depolymerisation defines a limiting activity represented by

$$
a_{\mathrm{MO}, \text { binary }}=\frac{\left(\mathrm{O}^{2-}\right)}{\left(\mathrm{O}^{2-}\right)+\left(\mathrm{SiO}_{2}\right)} \in\left[\left(\mathrm{SiO}_{2}\right) \leq\left(\mathrm{SiO}_{2}\right)_{\min }\right] .
$$

Thus, the molar abundances of the various oxygen species cannot be expected to be those of the original Toop-Samis model, especially when highly basic oxides are present in the liquid phase. In solving thermodynamic activity on a Temkin model basis, Toop and Samis $(1962 a, b)$ observed that the mean extension of the polymer chains is univocally defined by a «polymerzsation path» depicted in terms of mean numbers of silicon atoms per polymer unit $\bar{\nu}_{\mathrm{Si}_{\mathrm{i}}}=\left(N_{\mathrm{SiO}_{2}}\right) / \sum$ anions versus the stoichiometric ratio $\left(\mathrm{O}^{-}\right) /\left[\left(\mathrm{O}^{-}\right)+\left(\mathrm{O}^{-}\right)+\mathrm{Si}^{\mathrm{IV}}\right]$ ( $c f$. figs. 2 and 3 in Toop and Samis, 1962a). However, their further assumption - that a single polymerization path of general validity in the ternary system $\mathrm{CaO}-\mathrm{FeO}-\mathrm{SiO}_{2}$ may be proposed on the basis of viscosity data - cannot be shared, because a different reaction constant, $K_{18}$, pertains to each $\mathrm{MO}-\mathrm{SiO}_{2}$ system and, as the activity of the basic dissolved oxide MO is implicitly defined by the partial derivative of the Gibbs free energy of mixing at any point of the compositional space of interest, we have (Ottonello, 2001)

$$
\begin{aligned}
& \sum \text { anions }=\frac{\left(\mathrm{O}^{2-}\right)}{\exp \left\{\frac{\left[G_{\text {mixing }}+\left(1-\mathrm{X}_{\mathrm{MO}}\right) \frac{\partial G_{\text {mixing }}}{\partial \mathrm{X}_{\mathrm{MO}}}\right]}{R T}\right\}} \\
& \frac{\partial G_{\text {mixing }}}{\partial X_{\mathrm{MO}}}=\frac{1}{2} R T \ln K_{18} \frac{\partial\left(\mathrm{O}^{-}\right)}{\partial X_{\mathrm{MO}}}
\end{aligned}
$$

$\left(\mathrm{O}^{-}\right)=\frac{2 X_{\mathrm{MO}}-4+\left[\left(4-2 X_{\mathrm{MO}}\right)^{2}+\right.}{}$

$\frac{\left.-32\left(4 K_{\mathrm{P}}-1\right)\left(X_{\mathrm{MO}}^{2}-X_{\mathrm{MO}}\right)\right]^{\frac{1}{2}}}{8 K_{18}-2}$

$\left(\mathrm{O}^{0}\right)=\frac{4-4 \mathrm{X}_{\mathrm{MO}}-\left(\mathrm{O}^{-}\right)}{2}$

$\left(\mathrm{O}^{2-}\right)=\mathrm{X}_{\mathrm{MO}}-\frac{\left(\mathrm{O}^{-}\right)}{2}$

$\mathrm{Si}^{\mathrm{IV}}=\left(\mathrm{SiO}_{2}\right)$ 
where the terms in brackets denote the number of moles and $X_{\mathrm{MO}}$ is the molar fraction of a generic basic oxide $\mathrm{MO}$ in a $\mathrm{MO}-\mathrm{SiO}_{2}$ binary join.

Distinct «polymerization paths» relating the mean number of silicon atoms in the polymeric units $\left(\bar{\nu}_{\mathrm{Si}}\right)$ to the relative proportion of singly bonded oxygen in the unit $\left(\mathrm{O}^{-}\right) /\left[\left(\mathrm{O}^{-}\right)+\left(\mathrm{O}^{0}\right)+\mathrm{Si}^{\mathrm{IV}}\right]$ may be calculated for the two limiting binaries $\mathrm{CaO}-\mathrm{SiO}_{2}$ and $\mathrm{FeO}-\mathrm{SiO}_{2}$ on the basis of the system of eqs. ((3.15) to (3.20)). These paths differ substantially from the general path proposed over 40 years ago by Toop and Samis (1962a,b) and, more importantly, they differ greatly from each other.

The apparent complexity introduced by recognizing that each oxide imposes its own imprint on the extension of polymeric units turns

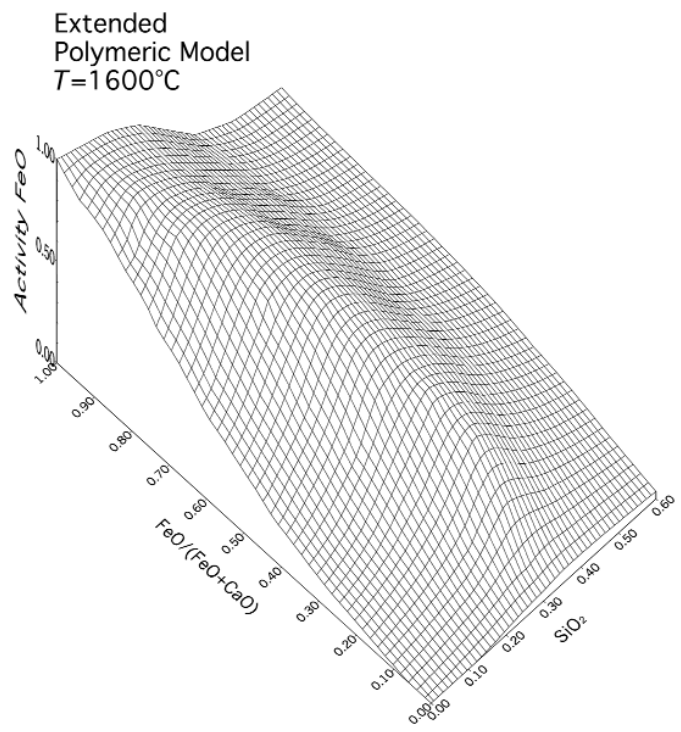

Fig. 2. FeO activity surface at $1600^{\circ} \mathrm{C}$ for liquid $\mathrm{FeO}-\mathrm{SiO}_{2}-\mathrm{CaO}$ ternary system. Graphic representation obtained by inverse-distance contouring of 9900 activity values generated by extended polymeric model, adopting, as limiting $K_{p}, 0.21$ for $\mathrm{FeO}-\mathrm{SiO}_{2}$ join and 0.002 for $\mathrm{CaO}-\mathrm{SiO}_{2}$ join, respectively. Assumed cationic interaction $W_{\mathrm{Ca}-\mathrm{Fe}}=-33 \mathrm{~kJ} / \mathrm{mol}$. Strain energy contributions included (from Ottonello, 2001, with modifications). out to be a powerful tool in deciphering the reactive properties of chemically complex melts. In fact, chemical interactions within the anion matrix of an $n$-component system are completely fixed by the interaction properties observed along the $n-1$ limiting binaries. As discussed elsewhere (Moretti and Ottonello, 2003), the intrinsic thermodynamic significance of this evidence is apparent in the Flood-Grjotheim thermodynamic cycle (Flood and Grjotheim, 1952). If $K_{P, A-F}, K_{P, B-F}$ are the polymerization constants valid along the limiting $A-F, B-F$ binaries, the polymerization constant of the extended Toop-Samis model turns out to be

$$
\ln K_{P}=N_{A^{v+}}^{\prime}+\ln K_{P, A-F}+N_{B^{0+}}^{\prime}+\ln K_{P, B-F}+\ldots
$$

The above equation states that, in a compositionally complex melt, network modifier cations $A, B$ of charge $v_{A}^{+}, v_{B}^{+}$interact with free oxygen $\mathrm{O}^{2-}$ (and hence with the polymeric units built up by network former $F$ ), with a simple proportionality described in terms of equivalent fractions (Flood and Grjotheim, 1952; Ottonello, 2001; Moretti and Ottonello, 2003)

$$
N_{A^{\nu+}}^{\prime}=\frac{\mathcal{V}_{A}^{+} n_{A}}{\mathcal{V}_{A}^{+} n_{A}+\mathcal{V}_{B}^{+} n_{B} \ldots}
$$

Application of the Flood-Grjotheim thermodynamic cycle to the $\mathrm{CaO}-\mathrm{FeO}-\mathrm{SiO}_{2}$ system satisfactorily reproduces the bulge in the thermodynamic activity of $\mathrm{FeO}$ experimentally observed long ago by Elliot (1955) (fig. 2). No high-order terms are necessary to reproduce the observed complex thermodynamic behavior, except for a topologically negligible interaction in the cation sublattice.

\subsection{Hybrid Polymeric Model}

In the Hybrid Polymeric Model (HPM) (Ottonello, 2001), the Gibbs free energy of mixing in binary melts is first subdivided into a (dominant) chemical Toop-Samis interaction term plus a (subordinate) elastic Hookian-like strain energy contribution term, as is observed in block copolymers (see later) 
$\Delta G_{\text {mixing }}=\Delta G_{\text {chemical }}+\Delta G_{\text {strain }}=\frac{\left(\mathrm{O}^{-}\right)}{2} R T \ln K_{18}+$ $+\frac{3 R T}{2 \bar{\nu}_{\mathrm{Si}}}\left(\frac{x}{a}\right)^{2}$

In eq. (3.23), $\bar{\nu}_{\mathrm{Si}}$ is the number of monomeric units of statistical length $a$ extended to a distance $x$, and the remaining symbols assume the usual thermodynamic significance. The elastic strain energy depends on bending term $x / a$ (which basically represents the effect of covalent bonding on the relative arrangement of monomers in the polymer chain). This term is arbitrarily expanded into a polynomial of type

$$
\frac{x}{a}=\chi_{1} \cdot N_{\mathrm{SiO}_{2}}+\chi_{2} \cdot N_{\mathrm{SiO}_{2}}^{2}+\chi_{3} \cdot N_{\mathrm{SiO}_{2}}^{3}+\chi_{4} \cdot N_{\mathrm{SiO}_{2}}^{4}+\ldots
$$

Strain coefficients $\chi_{1} \ldots \chi_{n}$ depict linear or second-order $T$ dependence. Since the polymerization path along any join is defined in terms of $\bar{\nu}_{\mathrm{Si}}$ versus $N_{\mathrm{SiO}_{2}}$, the strain energy calculated along a given binary at various $T$ conditions is intimately related to the extent of polymerization along any particular compositional path (i.e., the mean number of silicon atoms in the chain, $\left.\bar{v}_{\mathrm{Si}}\right)$. The existence of a maximum depolymerization limit and the approximate nature of the entropy terms depicted by the adopted parameterization of bending angles means that the problem cannot be solved exactly (more precise definition of entropic intermediate ordering effects on the bulk stability of the phase would require application of the self-consistent mean field theory - see later).

To overcome these complexities, Ottonello and Moretti (2004) modified eq. (3.23) as follows:

$$
\Delta G_{\text {mixing }}=\frac{\left(\mathrm{O}^{-}\right)}{2}\left(\Delta H_{18}-T \Delta S_{18}-T \eta\right) .
$$

In eq. (3.25), $S_{18}$ is the entropic contribution embodied in the Toop-Samis formulation of chemical interaction, and $\eta$ represents the strain contributions arising from the conformational arrangements of the various polyanions in the anion matrix. This term is again arbitrarily ex-

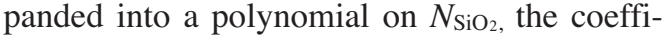
cients of which are inversely dependent on $T$

$$
\begin{aligned}
& \eta=\chi_{1}+\chi_{2} \cdot N_{\mathrm{SiO}_{2}}+\chi_{3} \cdot N_{\mathrm{SiO}_{2}}^{2}+\chi_{4} \cdot N_{\mathrm{SiO}_{2}}^{3} \\
& \chi_{1}=\chi_{0,1}+\chi_{1,1} / T .
\end{aligned}
$$

The new strain energy term $-T \eta$ is more akin to the modified-quasi-chemical formulation of the non-configurational entropy of mixing (Pelton and Blander, 1986) with respect to the original formulation (Ottonello, 2001) and allows better comparison of the two model energetics. Obviously, eq. (3.27) implies that, here, strain energy is not purely entropic, but embodies enthalpic and entropic sublattice interactions within the anion matrix which are not explained by the original Toop-Samis formulation. This amount of energy is far less than that arising from chemical interaction, and important only in quantifying the observed unmixing phenomena at high $\mathrm{SiO}_{2}$ content in the mixture (Ottonello, 2001).

Tables V and VI list the results of non-linear minimization calculations carried out to bring the modified quasi-chemical parameterization of Pelton et al. (1995) (tables III and IV) for binary $\mathrm{MO}-\mathrm{SiO}_{2}$ melts to an HPM formulation. The polymerization constants were first calculated for a single join at various $T$ conditions of interest in the specified $T$ range, and then regressed on an Arrhenian function of absolute T. Stemming from the obtained regression coefficients, there is no doubt that the adopted functional has precise thermodynamic significance which results in expansion (3.25) of the previous hybrid model.

The first striking evidence that arises from HPM treatment of interactions in $\mathrm{MO}-\mathrm{SiO}_{2}$ melts is the clear distinction between basic, amphoteric and acidic oxides. Basic oxides, which are essentially network modifiers in an $\mathrm{MO}-\mathrm{SiO}_{2}$ liquid, exhibit purely enthalpic contributions to chemical interactions in mixture, whereas acidic oxides (network formers) give rise to athermal behavior when mixed among themselves; and, clearly, amphoteric oxides exhibit both entropic and enthalpic contributions to chemical interactions in the melt phase.

If we now analyse the standard state enthalpic contribution to chemical interactions in $\mathrm{MO}-\mathrm{SiO}_{2}$ melts we note that they are simply re- 
Table V. Chemical interaction of HPM (from Ottonello and Moretti, 2004).

\begin{tabular}{cccccc}
\hline \hline Join & \multicolumn{2}{c}{$\ln K p=A / T+B$} & $R_{2}$ & $T$ range & Notes \\
& $A$ & $B$ & & $1{ }^{\circ} \mathrm{C}$ & \\
\hline $\mathrm{K}_{2} \mathrm{O}-\mathrm{SiO}_{2}$ & -31708 & 0 & - & $127-1527$ & $(1)$ \\
& -29540 & 0 & - & 1223 & $(2)$ \\
& -36967 & 0 & 0.9922 & $1500-1800$ & $(3)$ \\
$\mathrm{Na}_{2} \mathrm{O}-\mathrm{SiO}_{2}$ & -23336 & 0 & 0.9995 & $1000-1800$ & $(3)$ \\
$\mathrm{CaO}-\mathrm{SiO}_{2}$ & -15372 & 0 & 0.9958 & $1000-2000$ & $(4)$ \\
& -14807 & 0 & 0.9943 & $1000-2000$ & $(2)$ \\
$\mathrm{MgO}-\mathrm{SiO}_{2}$ & -9809.5 & 0 & 0.9955 & $1400-2000$ & $(3)$ \\
$\mathrm{ZnO}-\mathrm{SiO}_{2}$ & -6460.1 & 0 & - & $1400-2000$ & $(3)$ \\
$\mathrm{MnO}_{-} \mathrm{SiO}_{2}$ & -6183.8 & 0 & 0.9596 & $1000-2000$ & $(3)$ \\
& -5649.1 & 0 & - & 1600 & $(1)$ \\
$\mathrm{PbO}_{-} \mathrm{SiO}_{2}$ & -5330.0 & 0 & 0.5825 & $1000-1800$ & $(3)$ \\
& -4098.1 & 0 & - & 1273 & $(5)$ \\
$\mathrm{FeO}_{-}-\mathrm{SiO}_{2}$ & -3600.0 & 0 & 0.9973 & $1000-2000$ & $(4)$ \\
$\mathrm{Fe}_{2} \mathrm{O}_{3}-\mathrm{SiO}_{2}$ & 7569.5 & -7.2752 & 0.9350 & $1000-2000$ & $(5)$ \\
$\mathrm{TiO}_{2}-\mathrm{SiO}_{2}$ & 4667.3 & -3.2092 & 0.9107 & $1500-1900$ & $(3)$ \\
$\mathrm{ZrO}_{2}-\mathrm{SiOv}$ & 2685.9 & -6.382 & 0.9658 & $1400-2000$ & $(3)$ \\
$\mathrm{NiO}_{-} \mathrm{SiO}_{2}$ & 1507.7 & -1.7772 & 0.9825 & $1500-2000$ & $(3)$ \\
$\mathrm{Al}_{2} \mathrm{O}_{3}-\mathrm{SiO}_{2}$ & 0 & -1.4059 & - & $1000-2000$ & $(3)$ \\
$\mathrm{B}_{2} \mathrm{O}_{3}-\mathrm{SiO}_{2}$ & 0 & -1.0660 & - & $1000-2000$ & $(3)$ \\
\hline
\end{tabular}

(1) This work; (2) Ottonello and Moretti (2004), based on experiments of Eliezer et al. (1978); (3) Ottonello and Moretti (2004), based on modified quasi-chemical parameterization of Pelton et al. (1995); (4) Ottonello (2001); (5) Ottonello et al. (2001).

Table VI. Strain energy of HPM (from Ottonello and Moretti, 2004).

\begin{tabular}{|c|c|c|c|c|}
\hline \multirow{2}{*}{$\begin{array}{c}\text { Join } \\
\mathrm{K}_{2} \mathrm{O}-\mathrm{SiO}_{2}\end{array}$} & \multirow{2}{*}{$\begin{array}{c}\text { Parameterization } \\
\chi_{1} \\
\chi_{2} \\
\chi_{3} \\
\chi_{4}\end{array}$} & \multicolumn{2}{|c|}{$\begin{array}{l}\eta=\chi_{1}+\chi_{2} \cdot N_{\mathrm{SiO}_{2}}+\chi_{3} \cdot N_{\mathrm{SiO}_{2}}^{2}+\chi_{4} \cdot N_{\mathrm{SiO}_{2}}^{3} \\
\chi_{1}=\chi_{0,1}+\chi_{1,1} / T\end{array}$} & \multirow{2}{*}{$\begin{array}{c}\text { Notes } \\
(1)\end{array}$} \\
\hline & & $\begin{array}{r}-2.8681 \\
-79.501 \\
99.065 \\
10.499\end{array}$ & $\begin{array}{r}-1.6701 \mathrm{E}+5 \\
6.8787 \mathrm{E}+4 \\
1.5980 \mathrm{E}+5 \\
-1.9243 \mathrm{E}+3\end{array}$ & \\
\hline $\mathrm{Na}_{2} \mathrm{O}-\mathrm{SiO}_{2}$ & $\begin{array}{l}\chi_{1} \\
\chi_{2} \\
\chi_{3} \\
\chi_{4}\end{array}$ & $\begin{array}{r}-11.766 \\
39.776 \\
-141.53 \\
130.52\end{array}$ & $\begin{array}{r}-1.4559 \mathrm{E}+5 \\
2.2133 \mathrm{E}+5 \\
8.0710 \mathrm{E}+4 \\
-9.4332 \mathrm{E}+4\end{array}$ & (1) \\
\hline $\mathrm{CaO}-\mathrm{SiO}_{2}$ & $\begin{array}{l}\chi_{1} \\
\chi_{2} \\
\chi_{3} \\
\chi_{4}\end{array}$ & $\begin{array}{r}-1.7859 \\
31.535 \\
-171.49 \\
260.67\end{array}$ & $\begin{array}{r}-5.4644 \mathrm{E}+3 \\
-2.0262 \mathrm{E}+4 \\
2.8175 \mathrm{E}+5 \\
4.8533 \mathrm{E}+5\end{array}$ & (1) \\
\hline $\mathrm{MgO}-\mathrm{SiO}_{2}$ & $\begin{array}{l}\chi_{1} \\
\chi_{2}\end{array}$ & $\begin{array}{r}22.144 \\
-143.77\end{array}$ & $\begin{array}{r}-3.4756 \mathrm{E}+4 \\
2.2334 \mathrm{E}+5\end{array}$ & (1) \\
\hline
\end{tabular}


Table VI (continued).

\begin{tabular}{|c|c|c|c|c|}
\hline \multirow{2}{*}{$\begin{array}{c}\text { Join } \\
\mathrm{MgO}-\mathrm{SiO}_{2}\end{array}$} & \multirow{2}{*}{$\begin{array}{c}\text { Parameterization } \\
\chi_{3} \\
\chi_{4}\end{array}$} & \multicolumn{2}{|c|}{$\begin{array}{l}\eta=\chi_{1}+\chi_{2} \cdot N_{\mathrm{SiO}_{2}}+\chi_{3} \cdot N_{\mathrm{SiO}_{2}}^{2}+\chi_{4} \cdot N_{\mathrm{SiO}_{2}}^{3} \\
\chi_{1}=\chi_{0,1}+\chi_{1,1} / T\end{array}$} & \multirow[t]{2}{*}{ Notes } \\
\hline & & $\begin{array}{l}186.75 \\
173.75\end{array}$ & $\begin{array}{l}-3.4506 \mathrm{E}+5 \\
-2.7150 \mathrm{E}+4\end{array}$ & \\
\hline $\mathrm{ZnO}-\mathrm{SiO}_{2}$ & $\begin{array}{l}\chi_{1} \\
\chi_{2} \\
\chi_{3} \\
\chi_{4}\end{array}$ & $\begin{array}{r}-12.840 \\
-6.3910 \\
164.050 \\
-117.200\end{array}$ & $\begin{array}{r}3.5427 \mathrm{E}+4 \\
-1.4814 \mathrm{E}+5 \\
-6.1594 \mathrm{E}+4 \\
9.1512 \mathrm{E}+4\end{array}$ & (1) \\
\hline $\mathrm{MnO}-\mathrm{SiO}_{2}$ & $\begin{array}{l}\chi_{1} \\
\chi_{2} \\
\chi_{3} \\
\chi_{4}\end{array}$ & $\begin{array}{r}-7.3391 \\
-18.670 \\
92.477 \\
-23.422\end{array}$ & $\begin{array}{r}1.3047 \mathrm{E}+4 \\
3.5311 \mathrm{E}+4 \\
-1.8607 \mathrm{E}+5 \\
3.1079 \mathrm{E}+4\end{array}$ & (1) \\
\hline $\mathrm{PbO}-\mathrm{SiO}_{2}$ & $\begin{array}{l}\chi_{1} \\
\chi_{2} \\
\chi_{3} \\
\chi_{4}\end{array}$ & $\begin{array}{r}21.546 \\
-59.309 \\
132.23 \\
-58.665\end{array}$ & $\begin{array}{r}-3.5593 \mathrm{E}+4 \\
2.6842 \mathrm{E}+4 \\
-3.4732 \mathrm{E}+4 \\
-1.2444 \mathrm{E}+4\end{array}$ & (1) \\
\hline $\mathrm{FeO}-\mathrm{SiO}_{2}$ & $\begin{array}{l}\chi_{1} \\
\chi_{2} \\
\chi_{3} \\
\chi_{4}\end{array}$ & $\begin{array}{r}10.214 \\
-19.638 \\
67.340 \\
-37.220\end{array}$ & $\begin{array}{r}-2.6396 \mathrm{E}+4 \\
6.5843 \mathrm{E}+4 \\
-2.1477 \mathrm{E}+5 \\
1.2462 \mathrm{E}+5\end{array}$ & (2) \\
\hline $\mathrm{TiO}_{2}-\mathrm{SiO}_{2}$ & $\begin{array}{l}\chi_{1} \\
\chi_{2} \\
\chi_{3} \\
\chi_{4}\end{array}$ & $\begin{array}{r}-11.099 \\
-20.417 \\
7.2065 \\
30.269\end{array}$ & $\begin{array}{r}1.7398 \mathrm{E}+4 \\
-8.0525 \mathrm{E}+2 \\
2.5437 \mathrm{E}+4 \\
-4.3948 \mathrm{E}+4\end{array}$ & (1) \\
\hline $\mathrm{ZrO}_{2}-\mathrm{SiO}_{2}$ & $\begin{array}{l}\chi_{1} \\
\chi_{2} \\
\chi_{3} \\
\chi_{4}\end{array}$ & $\begin{array}{r}-15.898 \\
-18.918 \\
16.482 \\
14.203\end{array}$ & $\begin{array}{r}2.2410 \mathrm{E}+4 \\
-1.7096 \mathrm{E}+4 \\
-1.2937 \mathrm{E}+3 \\
-1.3222 \mathrm{E}+4\end{array}$ & (1) \\
\hline $\mathrm{NiO}-\mathrm{SiO}_{2}$ & $\begin{array}{l}\chi_{1} \\
\chi_{2} \\
\chi_{3} \\
\chi_{4}\end{array}$ & $\begin{array}{r}-3.2377 \\
21.656 \\
-49.883 \\
18.565\end{array}$ & $\begin{array}{r}2.7449 \mathrm{E}+3 \\
4.9178 \mathrm{E}+3 \\
-4.5618 \mathrm{E}+4 \\
5.9709 \mathrm{E}+4\end{array}$ & (1) \\
\hline $\mathrm{Al}_{2} \mathrm{O}_{3}-\mathrm{SiO}_{2}$ & $\begin{array}{l}\chi_{1} \\
\chi_{2} \\
\chi_{3} \\
\chi_{4}\end{array}$ & $\begin{array}{r}-3.7071 \\
26.748 \\
-64.828 \\
77.543\end{array}$ & $\begin{array}{r}-6.1142 \mathrm{E}+2 \\
-1.5242 \mathrm{E}+4 \\
4.2276 \mathrm{E}+4 \\
-7.0683 \mathrm{E}+4\end{array}$ & (1) \\
\hline $\mathrm{B}_{2} \mathrm{O}_{3}-\mathrm{SiO}_{2}$ & $\begin{array}{l}\chi_{1} \\
\chi_{2} \\
\chi_{3} \\
\chi_{4}\end{array}$ & $\begin{array}{r}0.0928 \\
11.819 \\
-41.175 \\
36.358\end{array}$ & $\begin{array}{r}-6.1472 \mathrm{E}+3 \\
7.9109 \mathrm{E}+3 \\
-2.0305 \mathrm{E}+4 \\
3.8358 \mathrm{E}+5\end{array}$ & (1) \\
\hline
\end{tabular}

(1) Based on modified quasi-chemical parameterization of Pelton et al. (1995); (2) based on modified quasichemical parameterization of Pelton and Blander (1986) and Pelton (pers. comm.).

lated to the atomistic properties of the central cation, like Pauling's electronegativity (Pauling, 1932, 1960) (fig. 3) or to spectroscopically derived magnitudes, such as differences in opti- cal basicity between metal oxide and silica (Duffy and Ingram, 1974; Gaskell, 1982; Sosinsky and Sommerville, 1986; Duffy, 1989, 1990; Ottonello et al., 2001) (fig. 4). 

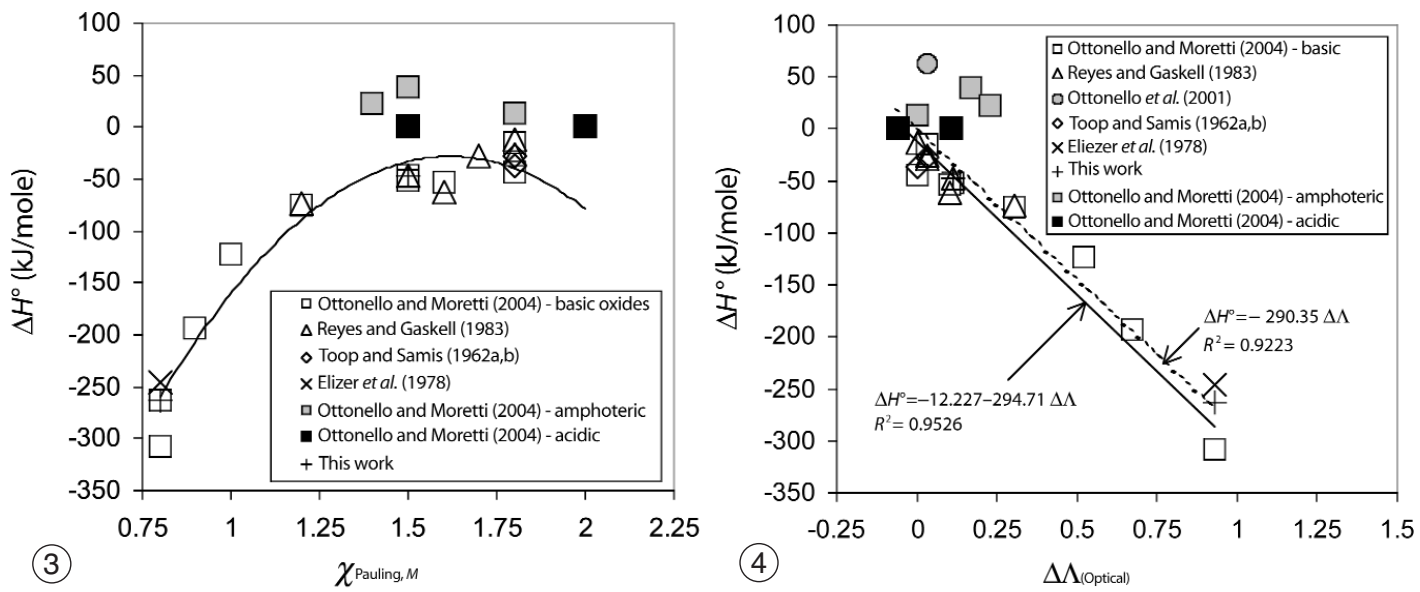

Fig. 3. Reaction enthalpy of equilibrium 2 plotted against Pauling's electronegativity of MO metal cation.

Fig. 4. Reaction enthalpy of equilibrium 2 plotted against difference in optical basicity of $\mathrm{MO}$ and $\mathrm{SiO}_{2}$. Adopted basicity values are those of column 6 in table A1 of Ottonello et al. (2001). Selection of other basicity values within uncertainty ranges (columns 1 to 6 in same table) do not greatly alter observed relations.

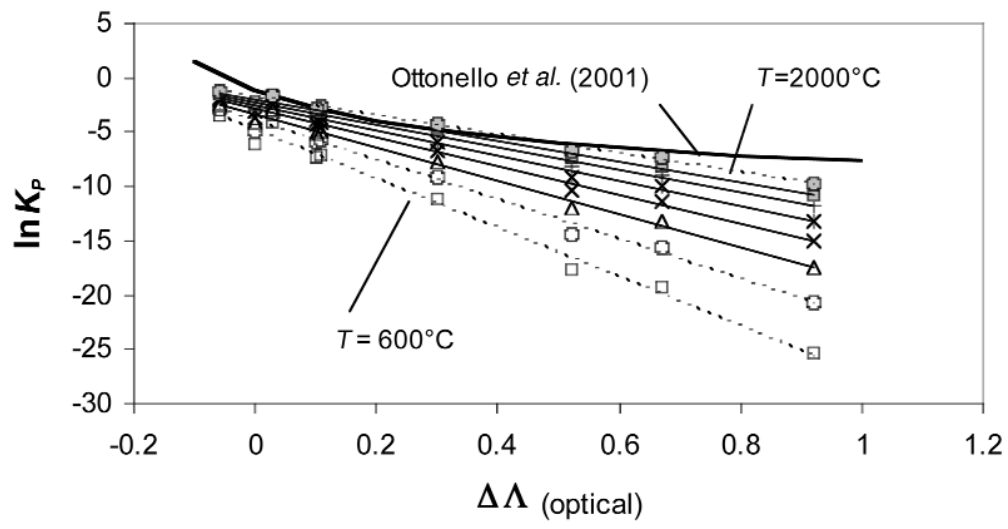

Fig. 5. Polymerization constant in $\mathrm{MO}-\mathrm{SiO}_{2}$ melts at various $T$ (with $\mathrm{MO}=$ basic oxide) plotted against difference in optical basicity of $\mathrm{MO}$ and $\mathrm{SiO}_{2}$ (adopted basicity values are those of column 6 in table A1 of Ottonello et al., 2001). Selection of other basicity values within uncertainty ranges (columns 1 to 6 in same table) do not greatly alter observed relations. Dashed lines: extrapolations at $T$ beyond $T$ limits investigated here for most basic oxides. Solid heavy line: functional form adopted by Ottonello et al. (2001) and Moretti and Ottonello (2003) when role of $T$ was still unclear.

The discrimination capability of optical basicity in terms of Lux-Flood basicity is even clearer when looking at polymerization constant $K_{P}$. As shown in fig. 5 , the relationship be- tween the natural logarithm of $K_{P}$ and the optical basicity difference is so evident as to allow it to be used as a predictive tool for the chemical interaction of basic oxides with silica. Each 
of the depicted straight-line dependencies in fig. 5 has a correlation coefficient $R^{2}=0.9985$, and their slopes and intercepts also exhibit a second-order dependence on absolute $T$.

\section{Configurational disorder in block copolymers}

Although there is no experimental evidence about the possibile formation of local block copolymeric structures in silicate melts or glasses, recent findings in this branch of materials science are also important for proper understanding of the unmixing phenomena which take place in $\mathrm{SiO}_{2}$-rich melts. The physics of microphase separation in block copolymers is understood in terms of an expanded Flory-Huggins model.

The Flory-Huggins model belongs to the class of quasi-chemical approaches. Flory himself defined his method as «a restricted variation of the quasi-chemical method used by Guggenheim» ( $c f$. Flory, 1953, p. 507). Basically, the main merit of the Flory-Huggins approach is to acknowledge that molar fractions are unsuitable chemical parameters whenever molecules differing greatly in size interact among themselves and with much smaller stoichiometric units in the melt phase. Volume fractions are thus substituted for molar fractions, based on the identity

$$
\phi_{i}=\frac{n_{i} v_{i}}{\sum_{i=1}^{k} n_{i} v_{i}}
$$

where $\phi_{i}$ is the volume fraction of the ith component in mixture, $n_{i}$ its molar amount, $v_{i}$ its partial molar volume, and the summation at the denominator is extended to all $k$ components in mixture.

Block copolymers are composed of two or more distinct blocks interconnected by linear or branched sequencing. The relative arrangements of the various blocks gives rise to an astonishing number of distinct configurations, which is responsible for the many useful and desirable properties exploited by the industry of rubberlike materials. The simplest architecture is the linear $A B$ diblock: a long sequence of type $A$ monomers covalently bonded to a chain of type $B$ monomers. The relative arrangements of the two blocks is characterized by «a fluidlike disorder on the molecular scale and a high degree of order at longer length scales» (Bates and Fredrickson, 1999).

In interpreting the structural and reactive properties of diblock copolymers, the following working assumptions are commonly made:

- Each molecule in the melt is composed of $v$ segments, $f A$-type and $(1-f) B$-type monomers.

- $A$ and $B$ segments represent a sufficient length of polymers such that they can be treated as Gaussian, where the internal conformational states of the segments produce a Hookian entropic penalty of stretching.

- The statistical length of a segment is its average RMS end-to-end length when no tension is applied, and is related to the effective spring constant.

- The average segment concentration is forced to be uniform (incompressibility constraint).

The interaction between $A$ and $B$ is described in terms of a $\chi_{A B}$ Flory-Huggins interaction parameter which, in units of thermal energy $k T$, is expressed as follows:

$$
\chi_{A B}=\frac{Z}{k T}\left[\varepsilon_{A B}-\frac{1}{2}\left(\varepsilon_{A A}+\varepsilon_{B B}\right)\right]
$$

where $Z$ is the number of nearest-neighbor monomers to a copolymer configuration cell (note the analogy with eq. (2.2)). If $\chi_{A B}$ is large, chemical interaction leads to a macroscopic segregation of $A$ and $B$ (binodal or spinodal decomposition). If instead $\chi_{A B}$ is sufficiently low, the thermodynamic forces driving separation are counterbalanced by entropic restoring contributions (i.e., "chain elasticity»; intimately connected to the covalent character of the bond but Hookian in nature) reflecting the requirement that, to keep the dissimilar $A$ and $B$ portions of each molecule apart, copolymers must adopt extended configurations (Bates and Fredrickson, 1999).

\subsection{Mean field approximation theories}

Helmholtz free energy $F$ (the incompressibility constraint) (Fredrickson et al., 1994, Fredrickson and Liu, 1995) is 


$$
F=\frac{k T}{4 \pi^{2}} \int_{0}^{\infty} q^{2} \ln \left[\phi_{A} S_{A}(q)+\phi_{B} S_{B}(q)\right] d q
$$

where $q$ is the reciprocal of the distance in the search space and $\mathrm{S}_{A}, \mathrm{~S}_{B}$ are form factors of the $A$ and $B$ block copolymers (Fredrickson et al., 1994; Fredrickson and Liu, 1995; Chen et al., $2000,2002)$. These «form factors» assume precise values depending on the extent and geometry of the block. For a linear chain $L$ of monomers of statistical Kuhn length b, the form factor is simply $\mathrm{S}_{L}=v_{L} g(x)$ with $v_{L}=$ degree of polymerization and

$$
\begin{gathered}
x=q^{2} b^{2} v_{L} / 6 \\
g(x)=\frac{2\left(\mathrm{e}^{-x}+x-1\right)}{x^{2}} .
\end{gathered}
$$

For branched polymers the form factors are more complex and are obtained by a combina-

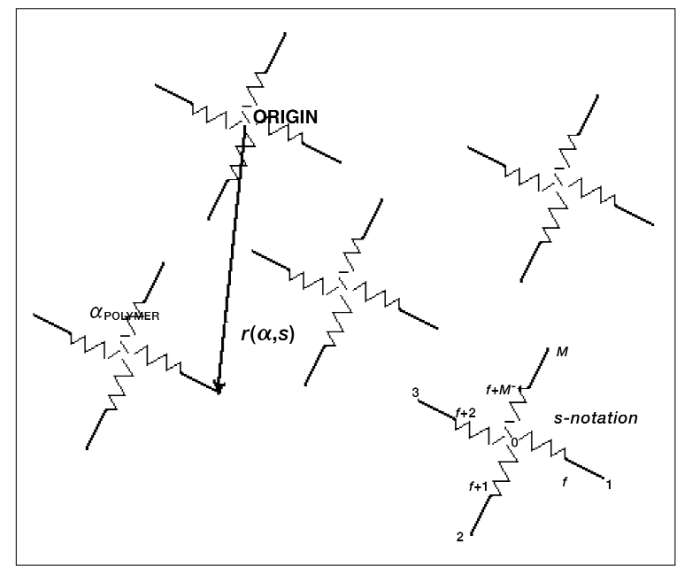

Fig. 6. Positional parametrization of starblock copolymers. Copolymer melt is composed of starblock copolymers made up of alternating blocks consisting of $f A$-type and ( $1-f) B$-type monomeric fractions. Positional vector defining relative position of various branches of the $i$ th copolymer is defined in terms of polymer number and branch number. Space function is piecewise continuous with discontinuities at integer values. tion of arm and backbone contributions (see Fredrickson et al., 1994, 1995, for appropriate treatment).

\subsection{Matsen-Schick self-consistent mean field method}

The spectral analysis developed by Matsen and Schick in a series of articles (Matsen and Schick, 1994a,b; Matsen and Bates, 1996; Matsen, 1998) is a powerful predictive tool in deciphering the complex microphase separation processes taking place in rubberlike materials. The theory stems from the Self-Consistent Mean Field (SCMF) treatment of Hong and Noolandi (1981). The form factor functionals are substituted here by space-occupation functions $\boldsymbol{r}_{\alpha}(s)$ describing the space curve occupied by the $\alpha$ th copolymer, and $s$ is the parameterization variable.

In a system of $n$ starblock copolymers composed of $M A B$ diblock arms joined together at a central core, $s=0$ at the core, $s=f$ at the $A B$ junction, and $s=1$ at the end of the first arm. In the second arm, $s$ is $1,1+f, 2$, and so on, until the end of the last arm, where $s=M$ is reached. The space occupation function is thus piecewise continuous with discontinuities at integer values (fig. 6).

The partition function for a system of $p$ polymers of polymerization $v$ and density $\rho_{0}$ is

$$
\begin{aligned}
& Q=\int D \boldsymbol{r}_{p} P\left[\boldsymbol{r}_{p} ; M+f\right] \delta\left[1-\hat{\boldsymbol{\phi}}_{A}-\hat{\boldsymbol{\phi}}_{B}\right] \times \\
& \times \exp \left\{-\frac{\rho_{0}}{\nu} \int d \boldsymbol{r} \chi_{A B} \nu \hat{\boldsymbol{\phi}}_{A}(\boldsymbol{r}) \hat{\boldsymbol{\phi}}_{B}(\boldsymbol{r})\right\} .
\end{aligned}
$$

$P$ is the probability density functional for a given curve (see eq. 2 in Matsen and Schick, $1994 \mathrm{a}$, for the approximate form adopted to describe $P) ; \hat{\phi}_{A}(\boldsymbol{r}), \hat{\phi}_{B}(\boldsymbol{r})$ are (dimensionless) $A$ and $B$ monomer density operators

$$
\begin{aligned}
& \hat{\phi}_{A}(\boldsymbol{r})=\frac{\nu}{\rho_{0}} \int_{0}^{M+f} d s \gamma(s) \cdot \delta\left[\boldsymbol{r}-\boldsymbol{r}_{p}(s)\right] \\
& \hat{\phi}_{B}(\boldsymbol{r})=\frac{\nu}{\rho_{0}} \int_{0}^{M+f} d s(1-\gamma(s)) \cdot \delta\left[\boldsymbol{r}-\boldsymbol{r}_{p}(s)\right]
\end{aligned}
$$


$\gamma(s)$ is a discontinuity function which attains a value of 1 when s corresponds to an $A$-monomer region and a value of 0 for a $B$-monomer region. The product of density operators in eq. (4.6) represents the various space-dependent interactions in the copolymer melt.

To render the problem mathematically tractable, the following steps are performed:

1) A Delta functional integral

$$
1=\int D \Phi_{A} \delta\left[\Phi_{A}-\hat{\phi}_{A}\right]
$$

allows the chain density operator to be replaced by segment volume fractions $\phi_{A}$.

2) Segment volume fractions are expanded as linear combinations of basis functions

$$
\phi_{A}(r)=\phi_{A, 1} f_{1}(r)+\phi_{2} f_{2}(r)+\phi_{A, 3} f_{3}(r)+\ldots
$$

3) Basis functions (Fourier transforms) possessing the symmetry of the phase in question are selected, i.e., for the gyroid phase with symmetry $Q_{I a \overline{3} d}$ (Matsen and Schick, 1994b)

$$
\begin{aligned}
& f_{1}(r)=1 \\
& f_{2}(r)=\sqrt{8 / 3}[\cos (X) \sin (Y) \sin (2 Z)+\cos (Y) \cdot \\
& \cdot \sin (Z) \sin (2 X)+\cos (Z) \sin (X) \sin (2 Y)] \\
& f_{3}(r)=\sqrt{4 / 3}[\cos (2 X) \cos (2 Y)+\cos (2 Y) \\
& \cdot \cos (2 Z)+\cos (2 Z) \cos (2 X)] \\
& f_{4}(r)=\ldots \\
& f_{5}(r)=\ldots
\end{aligned}
$$

with $X=2 \pi x / d$ and $d=$ size of the cubic (gyroid) unit cell.

Several passages yield SCMF equations, reducing the problem to that of average densities of the $A, B$ monomers at $\boldsymbol{r}$ in the ensemble of non-interacting polymers subject to self-consistent external fields ( $c f$. eqs. 11-15 in Matsen and Schick, 1994a).

In various configurational states, Helmholtz free energy $F=-k t \ln Q$ is computed, and the minimum value (saddle point), attained through steepest descent techniques, is selected as representative of the stable configuration.

\section{Discussion}

For the sake of clarity, the discussion concerning information arising from the thermodynamic treatment of binary silicate melt interactions is divided into three distinct sections: 1) insights into short- and medium-range structural arrangement; 2) chemical interaction and longrange disorder; 3 ) and inhomogeneity ranges.

\subsection{Short-and medium-range disorder: insights from the EPA}

In fig. 7 , the polymerization paths of the EPA for various binary joins overlap the discrete $\left(\bar{v}_{\mathrm{Si}}\right)-\left(\mathrm{O}^{-}\right) /\left[\left(\mathrm{O}^{-}\right)+\left(\mathrm{O}^{0}\right)+\mathrm{Si}^{\mathrm{IV}}\right]$ values for structural units thought to be present in silicate melts and glasses (Toop and Samis, 1962a,b). For comparative purposes, in the same plot, the consolute point in the high- $\mathrm{SiO}_{2}$ portion of each binary join also overlaps. This sort of plot is very informative, since the following evidence may be retrieved:

- In most joins, the melt is fully depolymerized over a large part of the compositional range.

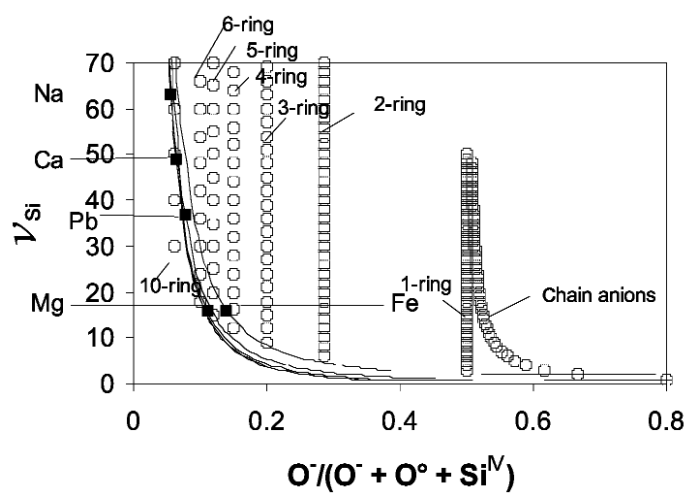

Fig. 7. Polymerization paths of EPA model compared with compositions of discrete polymeric units which may form in melt at various $\mathrm{SiO}_{2}$ contents. Adopted polymerization constants in constructing various curves are those of table V. Location of consolute points overlap, for comparative purposes. 
- Incipient decomposition (intrinsic instability) tendentially takes place when ring conformations change (e.g., 3-to-4 members, 4-to5 members, and so on).

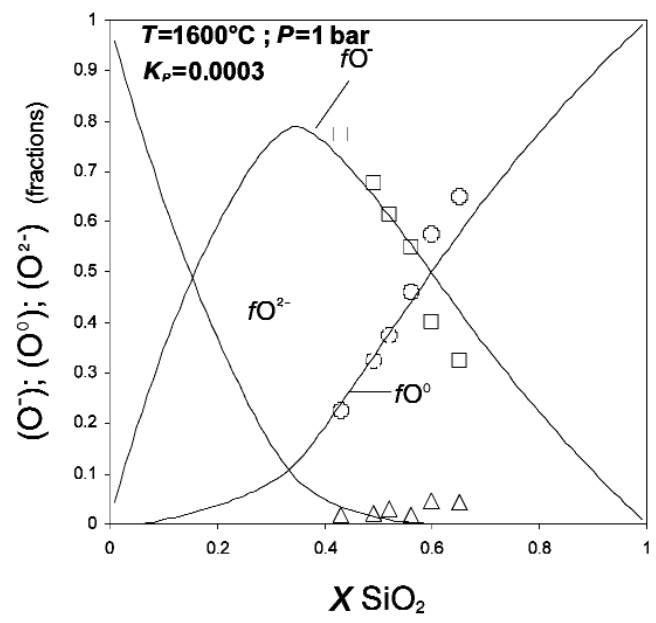

Fig. 8. XPS measurements of oxygen speciation in quenched $\mathrm{CaO}-\mathrm{SiO}_{2}$ melts (after Park and Rhee, 2001), compared with EPA predictions (Ottonello, 2001).
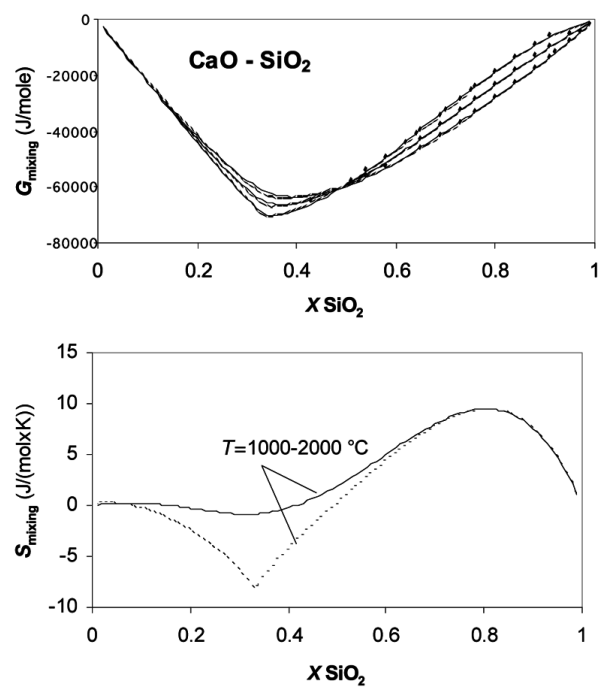

- Ring connectivity at the consolute point depends on the Lux-Flood basicity of the dissolved oxide (i.e., 4-rings-4-membered for $\mathrm{FeO}, 10$-rings-6-membered for $\mathrm{Na}_{2} \mathrm{O}$ ).

Figure 8 compares experimental evidence concerning the speciation of the various forms of oxygen present in a $\mathrm{CaO}-\mathrm{SiO}_{2}$ melt at $T=$ $=1600^{\circ} \mathrm{C}, P=1 \mathrm{bar}$, obtained by Park and Rhee (2001) by XPS ( $X$-ray Photoelectron Spectroscopy) with EPA predictions. This plot, besides experimentally confirming for the first time (as far as we know) the actual presence of oxide ions in the silicate melt, leaves few doubts about the soundness of the polymeric approach in depicting oxygen speciation. The fit between calculated and observed speciation is particularly satisfactory at low $\mathrm{SiO}_{2}$ amounts. At high silica content, the polymeric model appears to underestimate the degree of condensation of the polymeric units to some extent. This may be interpreted (in line with the preceding observations) as due to strain energy contribution effects (not accounted for in the model), which may stabilize high-membered rings in the vicinity of the two-phase region.
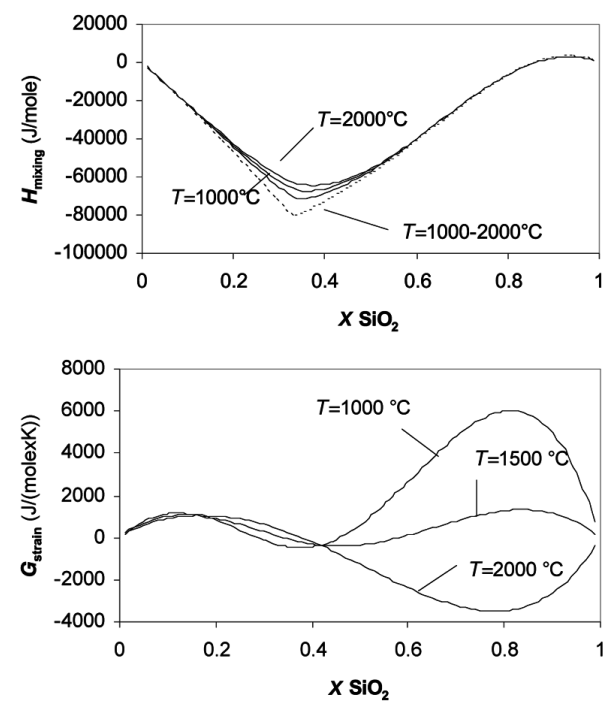

Fig. 9. HPM themodynamic parameterization of $\mathrm{CaO}-\mathrm{SiO}_{2}$ system (solid lines; Ottonello and Moretti, 2004), compared with estimates of modified quasi-chemical model (dashed lines; Pelton et al., 1995). 

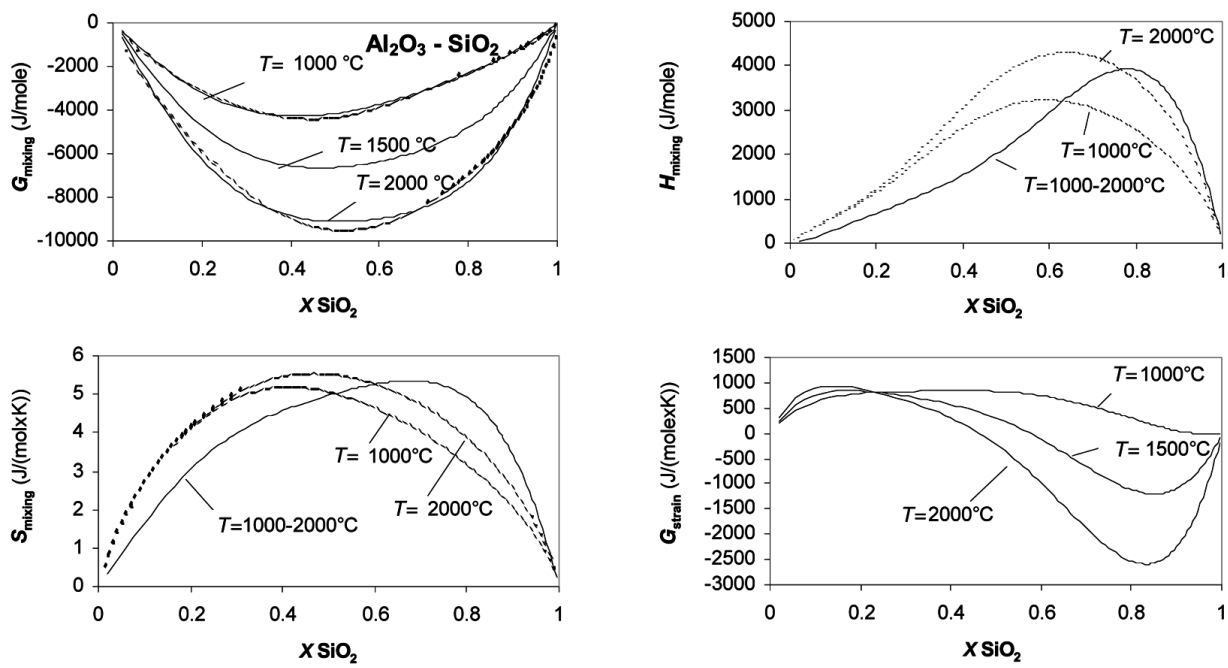

Fig. 10. HPM thermodynamic parameterization of $\mathrm{Al}_{2} \mathrm{O}_{3}-\mathrm{SiO}_{2}$ system (solid lines; Ottonello and Moretti, 2004), compared with estimates of modified quasi-chemical model (dashed lines; Pelton et al., 1995).

\subsection{Chemical interaction and long-range disorder}

As already mentioned, the HPM allows us to discriminate chemical (enthalpic + entropic) from elastic strain energy contributions to the bulk Gibbs free energy of mixing in $\mathrm{MO}-\mathrm{SiO}_{2}$ joins, and this discrimination is typical of the basic-amphotheric and acidic Lux-Flood behavior of MO oxides. The quasi-chemical to HPM conversion does not affect the bulk value of the Gibbs free energy of mixing of the join in question, although it does modify to some extent the relative magnitudes of enthalpic and entropic terms in the two models. As we see in fig. 9, for the $\mathrm{CaO}-\mathrm{SiO}_{2}$ join, entropic interactions observed for a Lux-Flood basic oxide arise entirely from elastic strain in the anion sublattice. Moreover, enthalpic chemical interactions are dominant, and the bulk strain energy contribution to the Gibbs free energy of mixing is far less and practically confined to the high- $\mathrm{SiO}_{2}$ portion of the system. Instead, when a Lux-Flood acidic oxide is dissolved in the silicate melt $\left(\mathrm{Al}_{2} \mathrm{O}_{3}-\mathrm{SiO}_{2}\right.$ join, fig. 10), although strain energy contributions are again confined to high $\mathrm{SiO}_{2}$ content, both enthalpic and entrop- ic chemical interaction terms are present over the entire compositional range, leading to an overall «regular» appearance of the bulk Gibbs free energy of mixing. Undoubtedly, $\mathrm{CaO}$ perturbs the long-range periodicity of the silicate network with local depolymerization effects whereas, at least apparently, «clusters» of overall $\mathrm{SiO}_{2}$ (tectosilicate) stoichiometry coexist stably with clusters of overall $\mathrm{Al}_{2} \mathrm{O}_{3}$ stoichiometry throughout the compositional range. Obviously, the picture is more complex for oxides with less definite Lux-Flood reactivity.

\subsection{Inhomogeneity ranges}

Although we can envisage the role of chemical interaction in determining (or modifying) short-, medium- and long-range periodicity in silicate melts and glasses, our appraisal is still insufficient to appreciate the complex phenomena which take place within the instability region. In the present author's opinion, better appraisal may be achieved by tentatively applying SCMF procedures to this topologically complex region.

Application of the Matsen-Schick theory to silicate polymer melts seems particularly prom- 


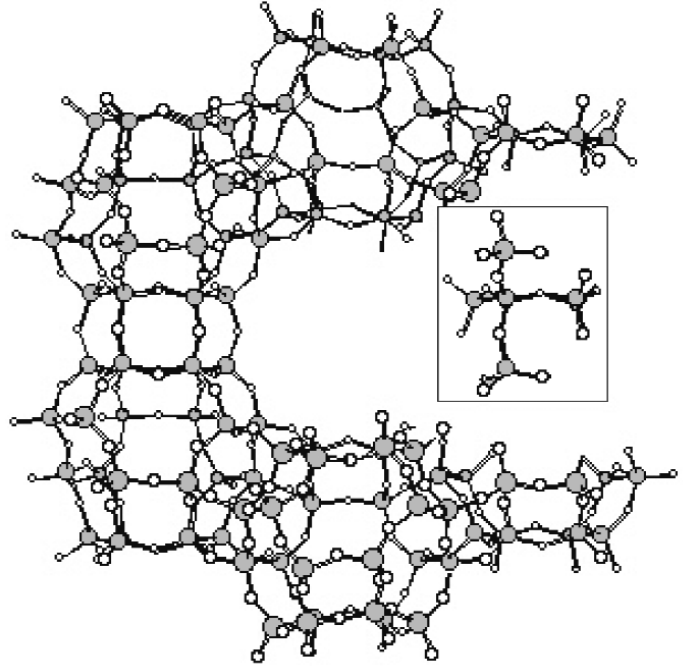

Fig. 11. Structure of an all-Si zeolite with «cages» of variable dimension and coordination number determined by $\mathrm{SiO}_{4}{ }^{4}$ network connectivity. Inset: irreducible representation of «starblock-like» copolymers composed of (identical) interacting $A-A$ monomers of same Kuhn length. Interaction parameter $\chi_{A A}$ is that arising from HPM treatment. Bending between adjacent polymers depends on value attained by $\chi_{A A}$ and determines medium-range order. Long-range periodicity is locally interrupted by depolymerization, and relative arrangement of starblock-like copolymers may contribute to structural instability (and consequent unmixing) through strain energy contributions.

ising. As anticipated in the introduction, the short- and medium-range periodicity of silicate melts and glasses locally resemble that of all-Si zeolites, with cationic «cages» of variable dimensions, depending on silicate network connectivity (fig. 11). In this sort of arrangement, it is still possible to isolate polymeric subunits (inset, fig. 11) of quasi-crystalline short- and medium-range order, but their relative positions perturb long-range periodicity. The following working assumptions are thus proposed, to render the problem feasible for SCMF treatment:

1) A simple, irreducible, $A$ - $A$ starblock-like representation of the network is selected as responsible for the short- and medium-range order observed in silicate melts and glasses (figs. 6 and 11).
2) It is assumed that, in the two-phase region, coexisting liquids locally mimic the structures of crystalline phases observed at the monotectic point.

3) Basic functions representative of the geometry of fictive intermixing $A-A$ starblocks (or $A-B$ starblocks, in the case of aluminosilicate melts) are assumed to be represented by the Fourier expansions of the spatial groups of the two solid phases at the monotectic point.

4) Calculations are conducted in terms of Gibbs free energy on the basis of HPM parameterization, after subtracting chemical interaction terms and adding $P V$ contributions.

\section{Conclusions}

Polymeric models provide an extremely useful tool in deciphering the structural and reactive properties of silicate melts and glasses. They not only establish the Lux-Flood character of the dissolved oxides through opportune conversion of existing quasi-chemical parameterization (Pelton and Blander, 1986; Pelton et al., 1995; Pelton, pers. comm.), but also discriminate the subordinate strain energy contributions to the Gibbs free energy of mixing from the dominant chemical interaction terms. This discrimination allows us to retrieve important information about the short-, medium- and long-range periodicity of this class of substances from thermodynamic analysis. However, the conceptual models developed for silicate melts and glasses are still insufficient to allow thorough appraisal of the complex phenomena which take place within the inhomogeneity range. It is thus suggested that a further important step may be made by applying SCMF theory and, particularly, Matsen-Schick spectral analysis, hitherto applied only to rubberlike materials.

\section{REFERENCES}

BATES, F.S. and G.H. FrEDRICKSON (1999): Block copolymers - designer soft materials, Phys. Today, 52 (2), 32-38.

BERNAL, J.D. (1959): A geometrical approach to the structure of liquids, Nature, 183, 141-147.

Brown, G.E. Jr., F. Farges and G. Calas (1995): X-ray 
scattering and $X$-ray spectroscopy studies of silicate melts, Rev. Mineral. Geochem., 32, 317-410.

Carraro, G. (1964): Physics of Non-Crystalline Solids (North-Holland Publishing Company, Amsterdam).

Chen, Y.Y., T.P. Lodge and F.S. BATES (2000): Entropically driven phase separation of highly branched/linear polyolefin blends, J. Polym. Sci. B, 38, 2965-2975.

CHEN, Y.Y., T.P. Lodge and F.S. BATES (2002): Influence of long-chain branching on the miscibility of poly(ethylene-r-ethylethylene) blends with different microstructures, J. Polym. Sci. B, 40, 466-477.

CripPS-Clark, C.J., R. SRIDHAR, J.H.E. JefFes and F.D. RICHARDSON (1974): Chain distribution and transition temperatures for phosphate glasses, in Physical Chemistry of Process Metallurgy, edted by J.H.E. JEFFES and R.J. TAIT (Inst. Mining Met.).

DUFFY, J.A. (1989): Use of refractivity data for obtaining optical basicity of transition metal oxides, Ironmaking Steelmaking, 16, 426-432.

Duffy, J.A. (1990): Bonding, Energy Levels and Bands (Longmans, London).

DuFFY, J.A. and M.D. INGRAM (1974): Optical basicity-IV: influence of electronegativity on the Lewis basicity and solvent properties of molten oxianion salts and glasses, J. Inorg. Nucl. Chem., 17, 1203-1206.

EliEZer, N., R.A. Howald, M. MARINKOviC and I. EliEZer (1978): Vapor pressure measurements, thermodynamic parameters and phase diagrams for the system potassium oxide - Silicon oxide at high temperatures, J. Phys. Chem., 82, 1021-1026.

ElliotT, J.F. (1955): Activities in the iron oxide-silica-lime system, Trans. Metall. Soc. AIME, 203, 485-488.

Evans, D.L. and S.V. KING (1966): Random network model of vitreous silica, Nature, 212, 1353-1354.

FINCHAM, C.J.B. and R.F. RICHARDSON (1954): The behaviour of sulfur in silicate and aluminate melts, Proc. $R$. Soc. London, A223, 40-61.

Flood, H. and K. GRJOTHEIM (1952): Thermodynamic calculation of slag equilibria, J. Iron Steel Inst., 171, 64-80.

FloRY, P.J. (1953): Principles of Polymer Chemistry (Cornell University Press, Ithaca-New York).

FowLER, R.H. and E.A. GUGGENHEIM (1939): Statistical Thermodynamics (Cambridge University Press, Cambridge).

FRASER, D.G. (1977): Thermodynamic properties of silicate melts, in Thermodynamics in Geology, edited by D.G. Fraser (D. Reidel Pub. Co., Dortrecht-Holland).

FREDRICKSON G.H. and A.J. LiU (1995): Design of miscible polyolefin copolymer blends, J. Polym. Sci. B, 33, 1203-1212.

FredRICKSON, G.H., A.J. LiU and F.S. BATES (1994): Entropic corrections to the Flory-Huggins theory of polymer blends: architectural and conformational effects, Macromolecules, 27, 2503-2511.

GASKELL, D.R. (1982) On the correlation between the distribution of phosphorus between slag metal and the theoretical optical basicity of the slag, Trans. Iron Steel Inst. Jpn., 22, 997-1000.

GAskell, P.H., M.C. ECKersley, A.C. BARNES and P. ChieuX (1991): Medium-range order in the cation distribution of a calcium silicate glass, Nature, 350, 675-677.

Greaves, G.N., A. Fontaine, P. Lagarde, D. Raux and S.J. GURMAN (1981): Local structure of silicate glasses, $\mathrm{Na}$ ture, 293, 611-616.
GugGEnHeIM, E.A.(1935) The statistical mechanics of regular solutions, Proc. R. Soc. London, A148, 304-312.

Hong, K.M. and J. NoOLANDi (1981): Theory of inhomogeneous multicomponent polymer systems, Macromolecules, 14, 727-736.

MAtSEN, M.W. (1998): Gyroid versus double-diamond in ABC triblock copolymer melts, J. Chem. Phys., 108, 785-796.

MAtSEN, M.W. and F.S. BAtes (1996): Unifying weak- and strong-segregation block copolymer theories, Macromolecules, 29, 1091-1098.

Matsen, M.W. and M. Schick (1994a): Microphase separation in starblock copolymer melts, Macromolecules, 27, 6761-6767.

MAtsen, M.W. and M. Schick (1994b): Stable and unstable phases of a diblock copolymer melt, Phys. Rev. Lett., 72, 2660-2663.

Meadowcroft, T.R. and F.D. Richardson (1965): Structural and thermodynamic aspects of phosphate glasses, Trans. Faraday Soc., 61, 54-70.

Moretti, R. and G. OtTonello (2003): A polymeric approach to the sulfide capacity of silicate slags and melts, Metall. Trans. B, 34B, 399-409.

OtTONELlo, G. (2001): Thermodynamic constraints arising from the polymeric approach to silicate slags: the system $\mathrm{CaO}-\mathrm{FeO}-\mathrm{SiO}_{2}$ as an example, J. Non-Cryst. Solids, 282, 72-85.

Ottonello, G. and R. Moretti (2004): Lux-Flood basicity of binary silicate melts, J. Phys. Chem. Solids, 65, 1609-1614.

Ottonello, G., R. Moretti, L. Marini and M. Vetuschi ZuCCOLINI (2001): Oxidation state of iron in silicate glasses and melts: a thermochemical model, Chem. Geol., 174, 157-179.

PAuling, L. (1932): Nature of the chemical bond, IV. The energy of single bonds and the relative electronegativity of atoms, J. Am. Chem. Soc., 54, 3570-3582.

PAuling, L. (1960): The Nature of the Chemical Bond (Cornell University Press, Ithaca-New York).

PARK, J. and P.C. RHEE (2001): Ionic properties of oxygen in slags, J. Non-Cryst. Solids, 282, 7-14.

Pelton, A.D. and M. Blander (1986): Thermodynamic analysis of ordered liquid solutions by a modified quasi-chemical approach - Application to silicate slags, Metall. Trans. B, 17B, 805-15.

Pelton, A.D., P. Wu and G. ERIKSSON (1995): Critical evaluation and optimization of the phase diagrams and thermodynamic properties of oxide systems, in Phase Diagrams for Ceramists, edited by R.S. RоTH (The American Ceramic Society).

REYES, R.A. and D.R. GASKELL (1983): The thermodynamic activity of $\mathrm{ZnO}$ in silicate melts, Metall. Trans. B, 14B, 725-731.

SosinSKy, D.J. and I.D. SommERVILLE (1986): The composition and temperature dependence of the sulfide capacity of metallurgical slags, Metall. Trans, 17B, 331-337.

Toop, G.W. and C.S. SAmis (1962a): Some new ionic concepts of silicate slags, Can. Metall. Q., 1, 129-152.

Toop, G.W. and C.S. SAMIS (1962b): Activities of ions in silicate melts, Trans. Metall. Soc. AIME, 224, 878-887.

WARREN, B.E. (1933): $X$-ray diffraction of vitreous silica, Zeits. Krist., 86, 349-358.

WARREN, B.E. (1937): X-ray determination of the structure 
of liquids and glass, J. Appl. Phys., 8, 645-654.

Warren, B.E., H. Krutter and O. Morningstar (1936)

Fourier analysis of $X$-ray patterns of vitreous $\mathrm{SiO}_{2}$ and $\mathrm{B}_{2} \mathrm{O}_{3}, J$. Am. Ceram. Soc., 19, 202-206.

WASEDA, Y. and H. SutTo (1977): The structure of molten alkali metal silicates, Trans. Iron Steel Inst. Jpn., 17, 82-91.

WASEDA, Y. and J.M. TogurI (1977) The structure of molten binary silicate systems $\mathrm{CaO}-\mathrm{SiO}_{2}$ and $\mathrm{MgO}-$ $\mathrm{SiO}_{2}$, Metall. Trans. B, 8B, 563-568.

WASEDA, Y. and J.M. TOGURI (1978): The structure of the molten $\mathrm{FeO}-\mathrm{SiO}_{2}$ system, Metall. Trans. B, 9B, 595-601. WASEDA, Y. and J.M. ToguRI (1990): Structure of silicate melts determined by $X$-ray diffraction, in Dynamic Processes of Material Transport and Transformation in the Earth's Interior, edited by F. MARUMO (Terra Scientific, Tokyo), 37-51.

ZACHARIASEN, W.H. (1932) The atomic arrangement in glass, J. Am. Chem. Soc., 54, 3841-3851.

ZaChariasen, W.H. (1933) The vitreous state, J. Chem. Phys., 3, 162-163. 